\title{
A Regularization SAA Scheme for a Stochastic Mathematical Program with Complementarity Constraints
}

\author{
Yu-xin Li, ${ }^{1}$ Jie Zhang, ${ }^{2}$ and Zun-quan $\mathrm{Xia}^{1}$ \\ ${ }^{1}$ Institute of ORCT, School of Mathematical Sciences, Dalian University of Technology, Dalian 116024, China \\ ${ }^{2}$ School of Mathematics, Liaoning Normal University, Dalian 116029, China \\ Correspondence should be addressed to Jie Zhang; zhangjie04212001@163.com
}

Received 29 May 2013; Accepted 3 November 2013; Published 10 February 2014

Academic Editor: Song Cen

Copyright (c) $2014 \mathrm{Yu}$-xin Li et al. This is an open access article distributed under the Creative Commons Attribution License, which permits unrestricted use, distribution, and reproduction in any medium, provided the original work is properly cited.

To reflect uncertain data in practical problems, stochastic versions of the mathematical program with complementarity constraints (MPCC) have drawn much attention in the recent literature. Our concern is the detailed analysis of convergence properties of a regularization sample average approximation (SAA) method for solving a stochastic mathematical program with complementarity constraints (SMPCC). The analysis of this regularization method is carried out in three steps: First, the almost sure convergence of optimal solutions of the regularized SAA problem to that of the true problem is established by the notion of epiconvergence in variational analysis. Second, under MPCC-MFCQ, which is weaker than MPCC-LICQ, we show that any accumulation point of Karash-Kuhn-Tucker points of the regularized SAA problem is almost surely a kind of stationary point of SMPCC as the sample size tends to infinity. Finally, some numerical results are reported to show the efficiency of the method proposed.

\section{Introduction}

Our concern in this paper is the following stochastic mathematical program with complementarity constraints (SMPCC):

$$
\begin{array}{ll}
\min & \mathbb{E}[f(z, \xi(\omega))] \\
\text { s.t. } & \mathbb{E}[g(z, \xi(\omega))] \leq 0, \\
& \mathbb{E}[h(z, \xi(\omega))]=0, \\
& 0 \leq \mathbb{E}[G(z, \xi(\omega))] \\
& \perp \mathbb{E}[H(z, \xi(\omega))] \geq 0,
\end{array}
$$

where $f: \mathfrak{R}^{n} \times \mathfrak{R}^{k} \rightarrow \mathfrak{R}, g: \mathfrak{R}^{n} \times \mathfrak{R}^{k} \rightarrow \mathfrak{R}^{p}$, $h: \mathfrak{R}^{n} \times \mathfrak{R}^{k} \rightarrow \mathfrak{R}^{q}, H: \mathfrak{R}^{n} \times \mathfrak{R}^{k} \rightarrow \mathfrak{R}^{m}$, and $G: \mathfrak{R}^{n} \times \mathfrak{R}^{k} \rightarrow \mathfrak{R}^{m}$ are random mappings; $\xi$ : $\Omega \rightarrow \Xi \subseteq \mathfrak{R}^{k}$ is a random vector defined on a probability space $(\Omega, \mathscr{F}, P)$; $\mathbb{E}$ denotes the mathematical expectation; the notation $\perp$ means "perpendicular." Throughout the paper, we assume that $\mathbb{E}[f(z, \xi(\omega))], \mathbb{E}[g(z, \xi(\omega))], \mathbb{E}[h(z, \xi(\omega))]$,
$\mathbb{E}[G(z, \xi(\omega))]$, and $\mathbb{E}[H(z, \xi(\omega))]$ are all well defined and finite for any $z \in \mathfrak{R}^{n}$. To ease the notation, we write $\xi(\omega)$ as $\xi$ and this should be distinguished from $\xi$ being a deterministic vector of $\Xi$ in a context.

The SMPCC (1) is a natural extension of deterministic mathematical program with complementarity constraints (MPCC) $[1,2]$, which have many applications in transportation [3] and communication networks [4], and so forth. There are many stochastic formulations of MPCC proposed in the recent years [3, 5-7]. Among these formulations, Birbil et al. [3] applied sample path method [8] to SMPCC (1).

In this paper, we are concerned with a numerical method for solving (1). Evidently, if the integral involved in the mathematical expectation of problem (1) can be evaluated either analytically or numerically, then problem (1) can be regarded as the usual MPCC problem and consequently it can be solved by existing numerical methods that are related. However, as shown in [9], in many situations, exact evaluation of the expected value in (1) for $x$ is either impossible or prohibitively expensive. Sample average approximation (SAA) method [8, 10 ] is suggested by many authors to handle such difficulty; see the recent works [11-15]. The basic idea of SAA is to generate 
an independent identically distributed (iid) sample $\xi^{1}, \ldots, \xi^{N}$ of $\xi$ and then approximate the expected value with sample average. In this context, let $\xi^{1}, \ldots, \xi^{N}$ be iid sample; then the SMPCC (1) is approximated by the following SAA problem:

$$
\begin{array}{ll}
\min & \widehat{f}^{N}(z) \\
\text { s.t. } & \widehat{g}^{N}(z) \leq 0, \quad \widehat{h}^{N}(z)=0, \\
& 0 \leq \widehat{G}^{N}(z) \perp \widehat{H}^{N}(z) \geq 0,
\end{array}
$$

where $\widehat{f}_{N}(z):=(1 / N) \sum_{i=1}^{N} f\left(z, \xi^{i}\right), \quad \widehat{g}_{N}(z) \quad:=$ $(1 / N) \sum_{i=1}^{N} g\left(z, \xi^{i}\right), \quad \widehat{h}_{N}(z) \quad:=\quad(1 / N) \sum_{i=1}^{N} h\left(z, \xi^{i}\right)$, $\widehat{G}_{N}(z):=(1 / N) \sum_{i=1}^{N} G\left(z, \xi^{i}\right), \widehat{H}_{N}(z):=(1 / N) \sum_{i=1}^{N} H\left(z, \xi^{i}\right)$ is the sample-average function of $f\left(z, \xi^{i}\right), g\left(z, \xi^{i}\right), h\left(z, \xi^{i}\right)$, $G\left(z, \xi^{i}\right)$ and $H\left(z, \xi^{i}\right)$ respectively. We refer to (1) as the true problem and (2) as the SAA problem to (1). Another critical problem for solving (1) is how to solve SAA problem (2) effectively. Since the Mangasarian-Fromovitz constraint qualification is violated at every feasible point of SAA problem (2) (see [16]), it is not appropriate to use standard nonlinear programming software to solve the SAA problem directly. The well-known regularization scheme [17], is a effective way to deal with this issue. That is, by replacing the complementarity constraint with a parameterized system of inequalities, the SAA problem is reformulated as follows:

$$
\begin{array}{ll}
\min & \widehat{f}^{N}(z) \\
\text { s.t. } & \widehat{g}^{N}(z) \leq 0, \quad \widehat{h}^{N}(z)=0, \\
& \widehat{G}^{N}(z) \geq 0, \quad \widehat{H}^{N}(z) \geq 0, \\
& \widehat{G}^{N}(z) \circ \widehat{H}^{N}(z) \leq t_{N} e,
\end{array}
$$

where $t_{N}>0$ is a parameter, "o" denotes the Hadamard product and $e \in \mathfrak{R}^{m}$ is a vector with components 1 . Then the SAA problem can be approximated by a smooth nonlinear programming (NLP) problem (3) when the parameter is sufficiently small. Consequently, a solution to true problem (1) can be obtained by solving a sequence of such regularized SAA problems.

In this paper, we focus on the detailed analysis of convergence properties of the regularized SAA problem (3) to the true problem (1) as the sample size tends to infinity. The main contributions of this paper can be summarized as follows: by the notion of epiconvergence in [18], we establish the almost sure convergence of optimal solutions of smoothed SAA problem as the sample size tends to infinity. Under MPCCMFCQ, we show that any accumulation point of KarashKuhn-Tucker points of the regularized SAA problem is a kind of stationary point almost surely. The obtained results can be seen an improvement of [17, Theorem 3.1] for solving SMPCC under weaker constraint qualification conditions. Moreover, under the MPCC strong second-order sufficient condition (MPCC-SSOSC) in [16], we investigate sufficient conditions under which the smoothed SAA problem possesses a KarashKuhn-Tucker point when the sample size is large enough, and the sequence of those points converges exponentially to a kind of stationary point of SMPCC almost surely as the sample size tends to infinity.

This paper is organized as follows: Section 2 gives preliminaries needed throughout the whole paper. In Sections 3 and 4 , we establish the almost sure convergence of optimal solutions and stationary points of the regularized SAA problem as the sample size tends to infinity respectively. In Section 5, existence and exponential convergence rate of stationary points of the regularized SAA problem are investigated. We also report some preliminary numerical results in Section 6.

\section{Preliminaries}

Throughout this paper we use the following notations. Let $\|\cdot\|$ denote the Euclidean norm of a vector or the Frobenius norm of a matrix. For a $m \times n$ matrix $A, A_{i j}$ denotes the element of the $i$ th row and $j$ th column of $A$. We use $I_{n}$ to denote the $n \times n$ identity matrix, $\mathbb{B}$ denotes the closed unite ball, and $\mathbb{B}(x, \delta)$ denotes the closed ball around $x$ of radius $\delta>0$. For a extended real-valued function $\varphi: \mathfrak{R}^{n} \rightarrow \mathfrak{R} \cup\{ \pm \infty\}$, epi $\varphi, \nabla \varphi(x)$, and $\nabla^{2} \varphi(x)$ denote their epigraph that is, the set $\{(x, \alpha) \mid \varphi(x) \leq \alpha\}$, the gradient of $\varphi$ at $x$, and the Hessian matrix of $\varphi$ at $x$, respectively. For a mapping $\phi: \mathfrak{R}^{n} \rightarrow \mathfrak{R}^{m}$, $\mathscr{J} \phi(x)$ denotes the Jacobian of $\phi$ at $x . \mathfrak{R}_{++}$stands for the positive real numbers.

In the following, we introduce some concepts of the convergence of set sequences and mapping sequences in [18] which will be used in the next section. Define

$$
\begin{gathered}
\mathcal{N}_{\infty}:=\{N \subseteq \mathbf{N} \mid \mathbf{N} \backslash N \text { finite }\} \\
\mathcal{N}_{\infty}^{\#}:=\{N \subseteq \mathbf{N} \mid N \text { infinite }\}
\end{gathered}
$$

where $\mathbf{N}$ denotes the set of all positive integer numbers.

Definition 1. For sets $C^{v}$ and $C$ in $\mathfrak{R}^{n}$ with $C$ closed, the sequence $\left\{C^{\nu}\right\}_{\nu \in \mathbf{N}}$ is said to converge to $C$ (written $C^{\nu} \rightarrow C$ ) if

$$
\limsup _{\nu \rightarrow \infty} C^{\nu} \subseteq C \subseteq \liminf _{\nu \rightarrow \infty} C^{\nu}
$$

with

$$
\begin{aligned}
& \limsup _{\nu \rightarrow \infty} C^{\nu}:=\left\{x \mid \exists N \in \mathcal{N}_{\infty}^{\#},\right. \\
&\left.\exists x^{\nu} \in C^{\nu}(\nu \in N) \text { such that } x^{\nu} \stackrel{N}{\rightarrow} x\right\}, \\
& \liminf _{\nu \rightarrow \infty} C^{\nu}:=\left\{x \mid \exists N \in \mathcal{N}_{\infty},\right. \\
&\left.\exists x^{\nu} \in C^{\nu}(\nu \in N) \text { such that } x^{\nu} \stackrel{N}{\rightarrow} x\right\} .
\end{aligned}
$$

The continuous properties of a set-valued mapping $S$ can be developed by the convergence of sets. 
Definition 2. A set-valued mapping $S: \mathfrak{R}^{n} \rightrightarrows \mathfrak{R}^{m}$ is continuous at $\bar{x}$, symbolized by $\lim _{x \rightarrow \bar{x}} S(x)=S(\bar{x})$, if

$$
\limsup _{x \rightarrow \bar{x}} S(x) \subseteq S(\bar{x}) \subseteq \liminf _{x \rightarrow \bar{x}} S(x) .
$$

Definition 3. Consider now a family of functions $f^{v}: \mathfrak{R}^{n} \rightarrow$ $\bar{\Re}$, where $\bar{\Re}=\mathfrak{R} \cup\{ \pm \infty\}$. One says that $f^{v}$ epiconverges to a function $f: \mathfrak{R}^{n} \rightarrow \bar{\Re}$ as $v \rightarrow \infty$ and is written as

$$
f=e-\lim _{\nu \rightarrow \infty} f^{\nu}
$$

if the sequence of sets epi $f^{\nu}$ converges to epi $f$ in $\Re^{n} \times \mathfrak{R}$ as $v \rightarrow \infty$.

Definition 4. Given a clos set $\Xi \subseteq \Re^{n}$ and a point $\bar{x} \in \Xi$. The cone

$$
\widehat{N}_{\Xi}(\bar{x}):=\left\{x^{*} \in \Re^{n} \mid \limsup _{\substack{\Xi \\ \rightarrow}} \frac{\left\langle x^{*}, x-\bar{x}\right\rangle}{\|x-\bar{x}\|} \leq 0\right\}
$$

is called the Fréchet normal cone to $\Xi$ at $\bar{x}$. Then the limiting normal cone (also known as Mordukhovich normal cone or basic normal cone) to $\Xi$ at $\bar{x}$ is defined by

$$
N_{\Xi}(\bar{x}):=\limsup _{x \stackrel{\Xi}{\rightarrow} \bar{x}} \widehat{N}_{\Xi}(x) .
$$

If $\Xi \subseteq \Re^{n}$ is a closed convex set, the limiting normal cone $N_{\Xi}(\bar{x})$ is the normal cone in the sense of convex analysis.

Next, we recall some basic concepts that are often employed in the literature on optimization problems with complementarity constraints.

Let $\bar{z}$ be a feasible point of problem (1) and for convenience we define the index sets

$$
\begin{aligned}
& I_{g}=\left\{i \in\{1,2, \ldots, p\}: \mathbb{E}\left[g_{i}(\bar{z}, \xi)\right]=0\right\}, \\
& \bar{\alpha}=\left\{i \in\{1,2, \ldots, m\}: \mathbb{E}\left[G_{i}(\bar{z}, \xi)\right]=0<\mathbb{E}\left[H_{i}(\bar{z}, \xi)\right]\right\}, \\
& \bar{\beta}=\left\{i \in\{1,2, \ldots, m\}: \mathbb{E}\left[G_{i}(\bar{z}, \xi)\right]=0=\mathbb{E}\left[H_{i}(\bar{z}, \xi)\right]\right\}, \\
& \bar{\gamma}=\left\{i \in\{1,2, \ldots, m\}: \mathbb{E}\left[G_{i}(\bar{z}, \xi)\right]>0=\mathbb{E}\left[H_{i}(\bar{z}, \xi)\right]\right\} .
\end{aligned}
$$

The constraint qualifications for SMPCC is as follows.

Definition 5. Assume $\mathbb{E}[g(\cdot, \xi)], \mathbb{E}[h(\cdot, \xi)], \mathbb{E}[G(\cdot, \xi)]$, and $\mathbb{E}[H(\cdot, \xi)]$ are continuously differentiable at $\bar{z}$. We say the MPCC Mangasarian-Fromovitz constraint qualification (MPCC-MFCQ) holds at $\bar{z}$ if the set of vectors

$$
\begin{aligned}
& \left\{\nabla \mathbb{E}\left[H_{i}(\bar{z}, \xi)\right] \nabla \mathbb{E}\left[h_{i}(\bar{z}, \xi)\right], i=1, \ldots, q ; \nabla \mathbb{E}\left[G_{i}(\bar{z}, \xi)\right],\right. \\
& \left.\quad i \in \bar{\alpha} \cup \bar{\beta} ; \nabla \mathbb{E}\left[H_{i}(\bar{z}, \xi)\right], i \in \bar{\beta} \cup \bar{\gamma}\right\}
\end{aligned}
$$

are linearly independent and there exists a nonzero vector $d \epsilon$ $\Re^{n}$ such that

$$
\begin{array}{ll}
\nabla \mathbb{E}\left[h_{i}(\bar{z}, \xi)\right]^{T} d=0 & i=1, \ldots, q, \\
\nabla \mathbb{E}\left[G_{i}(\bar{z}, \xi)\right]^{T} d=0 & i \in \bar{\alpha} \cup \bar{\beta}, \\
\nabla \mathbb{E}\left[H_{i}(\bar{z}, \xi)\right]^{T} d=0 & i \in \bar{\gamma} \cup \bar{\beta}, \\
\nabla \mathbb{E}\left[g_{i}(\bar{z}, \xi)\right]^{T} d<0 & i \in I_{g} .
\end{array}
$$

Definition 6. Assume $\mathbb{E}[g(\cdot, \xi)], \mathbb{E}[h(\cdot, \xi)], \mathbb{E}[G(\cdot, \xi)]$, and $\mathbb{E}[H(\cdot, \xi)]$ are continuously differentiable at $\bar{z}$. We say the MPCC linear independence constraint qualification (MPCCLICQ) holds at $\bar{z}$ if the set of vectors

$$
\begin{aligned}
& \left\{\nabla \mathbb{E}\left[g_{i}(\bar{z}, \xi)\right], i \in I_{g} ; \nabla \mathbb{E}\left[h_{i}(\bar{z}, \xi)\right], i=1, \ldots, q,\right. \\
& \left.\nabla \mathbb{E}\left[G_{i}(\bar{z}, \xi)\right], i \in \bar{\alpha} \cup \bar{\beta} ; \nabla \mathbb{E}\left[H_{i}(\bar{z}, \xi)\right], i \in \bar{\beta} \cup \bar{\gamma}\right\}
\end{aligned}
$$

are linearly independent.

As in [16], we use the following two stationarity concepts for SMPCC.

Definition 7. Assume $\bar{z}$ is a feasible point of SMPCC (1), $\mathbb{E}[g(\cdot, \xi)], \mathbb{E}[h(\cdot, \xi)], \mathbb{E}[G(\cdot, \xi)]$, and $\mathbb{E}[H(\cdot, \xi)]$ are continuously differentiable at $\bar{z}$. Suppose there exist vectors $\bar{\lambda} \in \mathfrak{R}^{\left|I_{g}\right|}$, $\bar{\mu} \in \mathfrak{R}^{q}, \bar{u} \in \mathfrak{R}^{|\alpha|+|\beta|}$, and $\bar{v} \in \mathfrak{R}^{|\beta|+|\gamma|}$ such that $\bar{z}$ satisfies the following conditions:

$$
\begin{aligned}
0= & \nabla \mathbb{E}[f(\bar{z}, \xi(\omega))]+\sum_{i \in I_{g}} \bar{\lambda}_{i} \nabla \mathbb{E}\left[g_{i}(\bar{z}, \xi)\right] \\
& +\sum_{i=1}^{q} \bar{\mu}_{i} \nabla \mathbb{E}\left[h_{i}(\bar{z}, \xi)\right]-\sum_{i \in \bar{\alpha} \cup \bar{\beta}} \bar{u}_{i} \nabla \mathbb{E}\left[G_{i}(\bar{z}, \xi)\right] \\
& -\sum_{i \in \bar{\beta} \cup \bar{\gamma}} \bar{v}_{i} \nabla \mathbb{E}\left[H_{i}(\bar{z}, \xi)\right] .
\end{aligned}
$$

(i) (C-stationary point) We call $\bar{z}$ a Clarke stationary point of (1) if $\bar{u}_{i} \bar{v}_{i} \geq 0, i \in \bar{\beta}$.

(ii) (S-stationary point) We call $\bar{z}$ a strongly stationary point of (1) if $\bar{u}_{i} \geq 0, \bar{v}_{i} \geq 0, i \in \bar{\beta}$.

The following upper level strict complementarity condition was used in [16] in the context of sensitivity analysis for MPCC.

Definition 8. We say that the upper level strict complementarity condition (ULSC) holds at $\bar{z}$ if $\bar{u}_{i}$ and $\bar{v}_{i}$, the multipliers correspondence to $\mathbb{E}\left[G_{i}(\bar{z}, \xi)\right]$, and $\mathbb{E}\left[H_{i}(\bar{z}, \xi)\right]$, respectively, satisfy $\bar{u}_{i} \bar{v}_{i} \neq 0$ for all $i \in \bar{\beta}$.

It is well known that a point $(\bar{x}, \bar{y})$ satisfies the lower level strict complementarity condition (LLSC) if $\mathbb{E}\left[G_{i}(\bar{z}, \xi)\right]+$ $\mathbb{E}\left[H_{i}(\bar{z}, \xi)\right]>0$ hold for all $i \in\{1, \ldots, m\}$, we can see from an example from [16] that ULSC condition is considerably 
weaker than the LLSC condition, and in practice, it may make more sense than the latter one.

We use the following second-order condition based on the MPCC-Lagrangian:

$$
\begin{aligned}
L(z, \lambda, \mu, u, v) & \\
= & \mathbb{E}[f(z, \xi)]+\sum_{i \in I_{g}} \lambda_{i} \mathbb{E}\left[g_{i}(z, \xi)\right] \\
& +\sum_{i=1}^{q} \mu_{i} \mathbb{E}\left[h_{i}(z, \xi)\right]-\sum_{i \in \bar{\alpha} \cup \bar{\beta}} u_{i} \mathbb{E}\left[G_{i}(z, \xi)\right] \\
& -\sum_{i \in \bar{\beta} \cup \bar{\gamma}} v_{i} \mathbb{E}\left[H_{i}(z, \xi)\right]
\end{aligned}
$$

of $(P)$.

Definition 9 (see [16]). Let $\bar{z}$ be a $S$-stationary point of (1) and $(\bar{\lambda}, \bar{\mu}, \bar{u}, \bar{v})$ is the corresponding multiplier at $\bar{z}$. Suppose $\mathbb{E}[g(\cdot, \xi)], \mathbb{E}[h(\cdot, \xi)], \mathbb{E}[G(\cdot, \xi)], \mathbb{E}[H(\cdot, \xi)]$, and $\mathbb{E}[f(\cdot, \xi)]$ are twice continuously differentiable at $\bar{z}$. We say that the MPCC strong second-order sufficient condition (MPCC-SSOSC) holds at $\bar{z}$ if

$$
d^{T} \nabla_{z}^{2} L(\bar{z}, \bar{\lambda}, \bar{\mu}, \bar{u}, \bar{v}) d>0
$$

for every nonvanishing $d$ with

$$
\begin{aligned}
& \nabla \mathbb{E}\left[g_{i}(\bar{z}, \xi)\right]^{T} d=0, \quad i \in I_{g}, \\
& \nabla \mathbb{E}\left[h_{i}(\bar{z}, \xi)\right]^{T} d=0, \quad i=1,2, \ldots, q, \\
& \nabla \mathbb{E}\left[G_{i}(\bar{z}, \xi)\right]^{T} d=0, \quad i \in \bar{\alpha}, \\
& \nabla \mathbb{E}\left[H_{i}(\bar{z}, \xi)\right]^{T} d=0, \quad i \in \bar{\gamma}, \\
& \min \left\{\nabla \mathbb{E}\left[G_{i}(\bar{z}, \xi)\right]^{T} d, \nabla \mathbb{E}\left[H_{i}(\bar{z}, \xi)\right]^{T} d\right\}=0, \\
& i \in \bar{\beta} .
\end{aligned}
$$

Assume $\bar{z}$ is a $S$-stationary point of (1) and $(\bar{\lambda}, \bar{\mu}, \bar{u}, \bar{v})$ is the corresponding multiplier. Then we know from $[16$, Theorem 7] that if MPCC-SSOSC holds at $\bar{z}$, it is a strict local minimizer of the SMPCC (1).

Throughout the paper, we assume the sample $\xi^{1}, \ldots, \xi^{N}$ of the random vector $\xi$ is iid and give the following assumptions to make (1) more clearly defined and to facilitate the analysis.

Assumption 10. The mapping $f(\cdot, \xi), G(\cdot, \xi), H(\cdot, \xi), g(\cdot, \xi)$, and $h(\cdot, \xi)$ are twice continuously differentiable on $\mathfrak{R}^{n}$ a.e. $\xi \in \Xi$.

Assumption 11. For any $\bar{z} \in \mathfrak{R}^{n}$, there exists a closed bounded neighborhood $D$ of $\bar{z}$ and a nonnegative measurable function $\kappa(\xi)$ such that $\mathbb{E}[\kappa(\xi)]<+\infty$ and

$$
\sup _{z \in D} \max \{\|\varrho(z, \xi)\|\} \leq \kappa(\xi)
$$

for all $\xi \in \Xi$, where $\varrho(z, \xi)$ is any element in the collection of functions $\left\{f(z, \xi), g(z, \xi), h(z, \xi), G(z, \xi), H(z, \xi), \mathscr{J}_{z} g(z, \xi)\right.$, $\nabla_{z} f(z, \xi), \mathscr{J}_{z} h(z, \xi), \mathscr{J}_{z} G(z, \xi)$, and $\left.\mathscr{J}_{z} H(z, \xi)\right\}$.

Assumption 12. For every $i \in\{1, \ldots, n\}$, the following properties hold ture.

(A1) For every $z \in \Re^{n}$, the moment generating function

$$
\mathscr{M}(t)_{i}:=\mathbb{E}\left[e^{\left(\left[\nabla_{z} f(z, \xi)\right]_{i}-\left[\mathbb{E}\left(\nabla_{z} f(z, \xi)\right)\right]_{i}\right)}\right]
$$

of random variable $\left[\nabla_{z} f(z, \xi)\right]_{i}-\left[\mathbb{E}\left(\nabla_{z} f(z, \xi)\right)\right]_{i}$ is finite valued for all $t$ in a neighborhood of zero.

(A2) There exists a measurable function $\kappa: \Xi \rightarrow \mathfrak{R}_{+}$such that

$$
\left\|\left[\nabla_{z} f(z, \xi)\right]_{i}-\left[\nabla_{z} f\left(z^{\prime}, \xi\right)\right]_{i}\right\| \leq \kappa(\xi)\left\|z-z^{\prime}\right\|
$$

for all $\xi \in \Xi$ and $z, z^{\prime} \in \mathfrak{R}^{n}$.

(A3) The moment generating $\mathscr{M}_{\kappa}(t)=\mathbb{E}\left[e^{t \kappa(\xi)}\right]$ of $\kappa(\xi)$ is finite valued for all $t$ in a neighborhood of zero.

Assumptions 10-12 are popularly used conditions for the analysis of SAA method for stochastic programming. Under Assumptions 10-11, we know from [10, Chapter 7] that $\mathbb{E}[f(z, \xi(\omega))]$ and $\mathbb{E}[G(z, \xi(\omega))]$ are twice continuously differentiable on $\mathfrak{R}^{n}$. In particular,

$$
\begin{aligned}
& \nabla \mathbb{E}[f(z, \xi(\omega))]=\mathbb{E}[\nabla f(z, \xi(\omega))], \\
& \mathscr{J} \mathbb{E}[G(z, \xi(\omega))]=\mathbb{E}[\mathscr{J} G(z, \xi(\omega))] .
\end{aligned}
$$

Assumption 12 is used to ensure exponential convergence rate of proposed regularization SAA method in Section 5.

The following results are directly from the Uniform Laws of Large Numbers in [10, Theorem 7.48].

Lemma 13. Let $\bar{z}$ be a feasible point of (1). Suppose that Assumptions 10-11 are satisfied; then we obtain

$$
\sup _{z \in D}\left\|\frac{1}{N} \sum_{i=1}^{N} \varrho(z, \xi)-\mathbb{E}[\varrho(z, \xi)]\right\| \longrightarrow 0 \quad \text { w.p.1, }
$$

where the set $D$ is a closed bounded neighborhood of $\bar{z}$ and $\varrho(z, \xi)$ is any element in the collection of functions $\left\{f(z, \xi), g(z, \xi), h(z, \xi), G(z, \xi), H(z, \xi), \mathscr{J}_{z} g(z, \xi), \nabla_{z} f(z, \xi)\right.$, $\mathscr{J}_{z} h(z, \xi), \mathscr{J}_{z} G(z, \xi)$, and $\left.\mathscr{J}_{z} H(z, \xi)\right\}$.

\section{Almost Sure Convergence of Optimal Solutions}

In this section, by the notion of epiconvergence in [18], we establish the almost convergence of optimal solutions of regularized SAA problem (3) to those of SMPCC (1) as the sample size tends to infinity. 
Let us introduce some notions:

$$
\begin{gathered}
\mathbb{Z}_{0}:=\left\{z \in \Re^{n}: \mathbb{E}[g(z, \xi)] \leq 0, \mathbb{E}[h(z, \xi)]=0,\right. \\
0 \leq \mathbb{E}[G(z, \xi)] \perp \mathbb{E}[H(z, \xi)] \geq 0\}, \\
\mathbb{Z}_{N}:=\left\{z \in \Re^{n}: \widehat{g}^{N}(z, \xi) \leq 0, \widehat{h}^{N}(z, \xi)=0,\right. \\
\left.\widehat{G}^{N}(z, \xi) \circ \widehat{H}^{N}(z, \xi) \leq t_{N} e\right\}, \\
\bar{f}^{N}(z):=\widehat{f}^{N}(z)+\delta_{\mathbb{Z}_{N}}(z), \\
\bar{f}(z):=\mathbb{E}[f(z, \xi)]+\delta_{\mathbb{Z}_{0}}(z), \\
\kappa_{0}:=\inf \left\{\mathbb{E}[f(z, \xi)]: z \in \mathbb{Z}_{0}\right\}, \\
S_{0}=\operatorname{argmin}\left\{\mathbb{E}[f(z, \xi)]: z \in \mathbb{Z}_{0}\right\}, \\
S_{N}:=\operatorname{argmin}\left\{\widehat{f}^{N}(z): z \in \mathbb{Z}_{N}\right\} .
\end{gathered}
$$

Now we give a conclusion about the almost sure convergence of the set $\mathbb{Z}_{N}$ as $N$ tends to infinity in the following proposition.

Proposition 14. Let $t_{N} \searrow 0$ as $N \rightarrow \infty$. Suppose Assumptions 10-11 hold. If MPCC-LICQ (Definition 6) holds for any $z \in \mathbb{Z}_{0}$, then

$$
\lim _{N \rightarrow \infty} \mathbb{Z}_{N}=\mathbb{Z}_{0} \quad \text { w.p.1. }
$$

Proof. We at first show that $\lim \sup _{N \rightarrow \infty} \mathbb{Z}_{N} \subseteq \mathbb{Z}_{0}$ w.p.1. It suffices to prove that for a sequence $\left\{z_{N}\right\}$ satisfying $z_{N} \in \mathbb{Z}_{N}$ for each $N$, if $z_{N}$ converges to $\bar{z}$ w.p.1 as $N \rightarrow \infty$, then $\bar{z} \epsilon$ $\mathbb{Z}_{0}$ w.p.1. Indeed, we know from the definition of $\mathbb{Z}_{N}$ that $z_{N}$ satisfies

$$
\begin{array}{ll}
\widehat{g}_{i}^{N}\left(z_{N}\right) \leq 0, & \widehat{h}_{j}^{N}\left(z_{N}\right)=0, \quad \widehat{H}_{k}^{N}\left(z_{N}\right) \geq 0, \\
\widehat{G}_{k}^{N}\left(z_{N}\right) \geq 0, & \widehat{H}_{k}^{N}\left(z_{N}\right) \widehat{G}_{k}^{N}\left(z_{N}\right) \leq t_{N}
\end{array}
$$

for $i=1,2, \ldots, p ; j=1,2, \ldots, q$; and $k=1,2, \ldots, m$, which, by Lemma 13 , means that $\bar{z} \in \mathbb{Z}_{0}$ w.p.1. Let

Let $\bar{z} \in \mathbb{Z}_{0}$. Next we show that $\bar{z} \in \liminf _{N \rightarrow \infty} \mathbb{Z}_{N}$ w.p.1.

$$
\Sigma(z)=\left\{z \in \mathfrak{R}^{n}: \Psi(z)-p=0\right\},
$$

where the mapping $\Psi: \mathfrak{R}^{n} \rightarrow \mathfrak{R}^{2 m+p+\left|I_{g}\right|}$ is defined by

$$
\Psi(p)=\left(\begin{array}{c}
\mathbb{E}\left[g_{i}(z, \xi)\right], \quad i \in I_{g} \\
\mathbb{E}\left[h_{i}(z, \xi)\right], i=1,2, \ldots, p \\
\mathbb{E}\left[G_{i}(z, \xi)\right], i \in \bar{\alpha} \cup \bar{\beta} \\
\mathbb{E}\left[H_{i}(z, \xi)\right], i \in \bar{\gamma} \cup \bar{\beta}
\end{array}\right) .
$$

Then $\bar{z} \in \Sigma(0) \cap Q_{0} \subseteq \mathbb{Z}_{0}$, where

$$
Q_{0}=\left\{\begin{aligned}
& \mathbb{E}\left[H_{i}(z, \xi)\right]>0, \quad i \in \bar{\alpha} \\
z \in \mathfrak{R}^{n}: & \mathbb{E}\left[G_{i}(z, \xi)\right]>0, \quad i \in \bar{\gamma} \\
& \mathbb{E}\left[g_{i}(z, \xi)\right]>0, \quad i \notin I_{g}
\end{aligned}\right\}
$$

Under MPCC-LICQ, $\Sigma(\cdot)$ has Aubin property [18] around $(0, \bar{z})$, which means that there exist constants $c>0, \varepsilon>0$, and $\delta>0$ such that

$$
\operatorname{dist}(z, \Sigma(p)) \leq c \operatorname{dist}\left(p, \Sigma^{-1}(z)\right)
$$

holds for $z \in \mathbb{B}(\bar{z}, \varepsilon)$ and $p \in \mathbb{B}(0, \delta)$. Therefore, for sufficiently small positive numbers $\varepsilon, \delta$, there exists a continuous function $z(\cdot): \mathbb{B}(0, \delta) \rightarrow \mathbb{B}(\bar{z}, \varepsilon)$ such that $z(0)=\bar{z}$ and for any $p \in \mathbb{B}(0, \delta)$,

$$
\Psi(z(p))-p=0
$$

Let

$$
p_{N}(z)=\left(\begin{array}{c}
\mathbb{E}\left[g_{i}(z, \xi)\right]-\widehat{g}_{i}^{N}(z), i \in I_{g} \\
\mathbb{E}[h(z, \xi)]-\widehat{h}^{N}(z) \\
\mathbb{E}\left[G_{i}(z, \xi)\right]-\widehat{G}_{i}^{N}(z), i \in \bar{\alpha} \\
\mathbb{E}\left[G_{i}(z, \xi)\right]-\widehat{G}_{i}^{N}(z)+\sqrt{t_{N}}, i \in \bar{\beta} \\
\mathbb{E}\left[H_{i}(z, \xi)\right]-\widehat{H}_{i}^{N}(z), i \in \bar{\gamma} \\
\mathbb{E}\left[H_{i}(z, \xi)\right]-\widehat{H}_{i}^{N}(z)+\sqrt{t_{N}}, i \in \bar{\beta}
\end{array}\right) .
$$

Then, by Lemma 13, we have for $N$ large enough

$$
\max _{z \in \mathbb{B}(\bar{z}, \varepsilon)}\left\|p_{N}(z)\right\|<\delta \quad \text { w.p.1 }
$$

and for any $z \in \mathbb{B}(\bar{z}, \varepsilon)$,

$$
\left\|z\left(p_{N}(z)\right)-\bar{z}\right\| \leq \varepsilon \quad \text { w.p.1. }
$$

Define a function

$$
\begin{gathered}
\varphi_{N}: \mathbb{B}(\bar{z}, \varepsilon) \longrightarrow \mathbb{B}(\bar{z}, \varepsilon) \\
z \longmapsto z\left(p_{N}(z)\right) .
\end{gathered}
$$

This is a continuous mapping from the compact convex set $\mathbb{B}(\bar{z}, \varepsilon)$ to itself. By Brouwer's fixed theorem, $\varphi_{N}$ has a fixed point. Hence, there exists a vector $z_{N} \in \mathbb{B}(\bar{z}, \varepsilon)$ w.p.1 such that $z_{N}=\varphi_{N}\left(z_{N}\right)=z\left(p_{N}\left(z_{N}\right)\right)$. Therefore, we have from (31) that

$$
0=\Psi\left(z\left(p_{N}\left(z_{N}\right)\right)\right)-p_{N}\left(z_{N}\right) .
$$

That is, $z_{N} \in \mathbb{P}_{0}^{N}$, w.p.1, where

$$
\mathbb{P}_{0}^{N}=\left\{\begin{array}{cc} 
& \widehat{g}_{i}^{N}(z)=0, i \in I_{g} \\
& \widehat{h}^{N}(z)=0 \\
z \in \Re^{n}: & \widehat{G}_{i}^{N}(z)=0, i \in \bar{\alpha} \\
& \widehat{G}_{i}^{N}(z)=\sqrt{t_{N}}, i \in \bar{\beta} \\
& \widehat{H}_{i}^{N}(z)=0, i \in \bar{\gamma} \\
& \widehat{H}_{i}^{N}(z)=\sqrt{t_{N}}, i \in \bar{\beta}
\end{array}\right\} .
$$

By Lemma 13, we obtain for sufficiently large $N, z_{N} \in Q_{N}$ due to $\bar{z} \in Q_{0}$, where

$$
Q_{N}=\left\{\begin{array}{ll} 
& \widehat{H}_{i}^{N}(z)>0, i \in \bar{\alpha} \\
z \in \Re^{n}: & \widehat{G}_{i}^{N}(z)>0, i \in \bar{\gamma} \\
& \widehat{g}_{i}^{N}(z)>0, i \notin I_{g}
\end{array}\right\},
$$

which means that $z_{N} \in Q_{N} \cap \mathbb{P}_{0}^{N} \subseteq \mathbb{Z}_{N}$. As a result, $\bar{z}$ belongs to $\lim \inf _{N \rightarrow \infty} \mathbb{Z}_{N}$ w.p.1 because of the almost sure convergence of $z_{N}$ to $\bar{z}$ as $N \rightarrow \infty$. We complete the proof. 
By Definition 3, similarly to the proof of [15, Lemma 4.3], we obtain the following lemma.

Lemma 15. Under the conditions of Proposition 14, we have

$$
e-\lim _{N \rightarrow \infty} \bar{f}^{N}=\bar{f} \quad \text { w.p.1. }
$$

The following result is directly from [18, Theorem 7.31]

Theorem 16. Suppose $z_{N}$ solves (3) for each $N$ and $\bar{z}$ is almost surely an accumulate point of the sequence $\left\{z_{N}\right\}$. If the conditions in Proposition 14 hold and $\kappa_{0}$ is finite, then $\bar{z}$ is almost surely an optimal solution of the true problem (1).

\section{Almost Sure Convergence of Stationary Points}

In practice, finding a global minimizer might be difficult and in some cases we might just find a stationary point. As a result, we want to know whether or not an accumulation point of the sequence of stationary points of regularized SAA problem (3) is almost surely a kind of stationary point of SMPCC (1).

Notice that (3) is a standard nonlinear programming with smooth constraints. If $z_{N}$ is a local optimal solution of the regularized SAA problem (3), then under some constraint qualifications, $z_{N}$ is a stationary point of (3); namely, there exists Lagrange multipliers $\lambda_{N} \in \mathfrak{R}^{p}, \mu_{N} \in \mathfrak{R}^{q}, a_{N} \in$ $\mathfrak{R}^{m}, b_{N} \in \mathfrak{R}^{m}$, and $\delta_{N} \in \mathfrak{R}^{m}$ such that the vector $\left(z_{N}, \lambda_{N}, \mu_{N}, a_{N}, b_{N}, \delta_{N}\right)$ satisfies the following Karash-KuhnTucker (KKT) condition for problem (3):

$$
\begin{aligned}
0= & \nabla \widehat{f}^{N}\left(z_{N}\right)+\mathscr{J} \widehat{g}^{N}\left(z_{N}\right)^{T} \lambda_{N}+\mathscr{J} \widehat{h}^{N}\left(z_{N}\right)^{T} \mu_{N} \\
& +\mathscr{J} \widehat{G}^{N}\left(z_{N}\right)^{T} a_{N}+\mathscr{J} \widehat{H}^{N}\left(z_{N}\right)^{T} b_{N} \\
& +\mathscr{J} \widehat{\Phi}^{N}\left(z_{N}\right)^{T} \delta_{N}
\end{aligned}
$$

with

$$
\begin{gathered}
0 \leq \lambda_{N} \perp \widehat{g}_{i}^{N}\left(z_{N}\right) \leq 0, \\
\widehat{\Phi}^{N}(z)=\widehat{H}_{i}^{N}(z) \circ \widehat{G}_{i}^{N}(z)-t_{N} e, \\
0 \geq \widehat{\Phi}^{N}\left(z_{N}\right) \perp \delta_{N} \geq 0, \\
0 \geq b_{N} \perp \widehat{H}^{N}\left(z_{N}\right) \geq 0, \\
0 \geq a_{N} \perp \widehat{G}^{N}\left(z_{N}\right) \geq 0 .
\end{gathered}
$$

We now prove the almost sure convergence of the regularization SAA method for SMPCC (1).

Theorem 17. Suppose Assumptions 10-11 hold. Let $t_{N} \searrow 0$ and let $z_{N}$ be a stationary point of problem (3). If the sequence $\left\{z_{N}\right\}$ converges to $\bar{z}$ w.p.1 as $N \rightarrow \infty$ and MPCC-MFCQ (Definition 5) holds at $\bar{z}$, then the following statements hold: (i) $\bar{z}$ is a C-stationary point of SMPCC (1) almost surely.

(ii) If, in addition, the multipliers $u_{i}=0$ and $v_{i}=0$ for all $i \in \beta \cap J_{0}$, where

$$
\begin{gathered}
J_{0}=\left\{i \in\{1,2, \ldots, m\}: \widehat{G}_{i}^{N}\left(z_{N}\right) \widehat{H}_{i}^{N}\left(z_{N}\right)\right. \\
\left.=t_{N} \text { for infinitely many } N\right\} \\
u_{i}=\lim _{N \rightarrow \infty}\left(\delta_{N}\right)_{i} \widehat{G}_{i}^{N}\left(z_{N}\right), \\
v_{i}=\lim _{N \rightarrow \infty}\left(\delta_{N}\right)_{i} \widehat{H}_{i}^{N}\left(z_{N}\right), \quad i \in J_{0},
\end{gathered}
$$

then $\bar{z}$ is a S-stationary point of SMPCC (1) almost surely.

Proof. Since $z_{N}$ is a stationary point of problem (3), there exist multipliers $\lambda_{N} \in \mathfrak{R}^{p}, \mu_{N} \in \mathfrak{R}^{q}, a_{N} \in \mathfrak{R}^{m}, b_{N} \in \mathfrak{R}^{m}$, and $\delta_{N} \in \mathfrak{R}^{m}$ such that

$$
\begin{aligned}
0= & \nabla \widehat{f}^{N}\left(z_{N}\right)+\mathscr{J} \widehat{g}^{N}\left(z_{N}\right)^{T} \lambda_{N}+\mathscr{J} \widehat{h}^{N}\left(z_{N}\right)^{T} \mu_{N} \\
& +\mathscr{J} \widehat{G}^{N}\left(z_{N}\right)^{T} a_{N}+\mathscr{J} \widehat{H}^{N}\left(z_{N}\right)^{T} b_{N} \\
& +\left(\begin{array}{c}
\left(\nabla \widehat{G}_{1}^{N}\left(z_{N}\right) \widehat{H}_{1}^{N}\left(z_{N}\right)+\nabla \widehat{H}_{1}^{N}\left(z_{N}\right) \widehat{G}_{1}^{N}\left(z_{N}\right)\right)^{T} \\
\vdots \\
\left(\nabla \widehat{G}_{1}^{N}\left(z_{N}\right) \widehat{H}_{m}^{N}\left(z_{N}\right)+\nabla \widehat{H}_{1}^{N}\left(z_{N}\right) \widehat{G}_{m}^{N}\left(z_{N}\right)\right)^{T}
\end{array}\right) \delta_{N}
\end{aligned}
$$

with

$$
\begin{gathered}
\left(\lambda_{N}\right)_{i} \geq 0, \quad \widehat{g}_{i}^{N}\left(z_{N}\right)\left(\lambda_{N}\right)_{i}=0, \quad i=1, \ldots, p, \\
\left(\widehat{H}_{i}^{N}\left(z_{N}\right) \widehat{G}_{i}^{N}\left(z_{N}\right)-t_{N}\right)\left(\delta_{N}\right)_{i}=0, \quad i=1, \ldots, m, \\
\left(a_{N}\right)_{i} \leq 0, \quad\left(b_{N}\right)_{i} \leq 0, \quad \widehat{H}_{i}^{N}\left(z_{N}\right)\left(b_{N}\right)_{i}=0, \\
\widehat{G}_{i}^{N}\left(z_{N}\right)\left(a_{N}\right)_{i}=0, \quad i=1,2, \ldots, m .
\end{gathered}
$$

Then (43) can be reformulated as

$$
\begin{aligned}
& -\nabla \widehat{f}^{N}\left(z_{N}\right) \\
& \left(\begin{array}{c}
\mathscr{J} \widehat{g}^{N}\left(z_{N}\right) \\
\mathscr{J} \widehat{h}^{N}\left(z_{N}\right) \\
\nabla \widehat{G}_{i}^{N}\left(z_{N}\right)^{T}, i \in J_{0}^{c} \\
\nabla \widehat{G}_{i}^{N}\left(z_{N}\right)^{T}+\frac{\widehat{G}_{i}^{N}\left(z_{N}\right)}{\widehat{H}_{i}^{N}\left(z_{N}\right)} \nabla \widehat{H}_{i}^{N}\left(z_{N}\right)^{T}, i \in J_{0} \cap \bar{\alpha} \\
\nabla \widehat{G}_{i}^{N}\left(z_{N}\right)^{T}, i \in J_{0} \cap \bar{\beta} \\
\nabla \widehat{H}_{i}^{N}\left(z_{N}\right)^{T}, i \in J_{0}^{c} \\
\nabla \widehat{H}_{i}^{N}\left(z_{N}\right)^{T}+\frac{\widehat{H}_{i}^{N}\left(z_{N}\right)}{\widehat{G}_{i}^{N}\left(z_{N}\right)} \nabla \widehat{G}_{i}^{N}\left(z_{N}\right)^{T}, i \in J_{0} \cap \bar{\gamma} \\
\nabla \widehat{H}_{i}^{N}\left(z_{N}\right)^{T}, i \in J_{0} \cap \bar{\beta}
\end{array}\right)^{T}
\end{aligned}
$$


with

$$
\begin{aligned}
& \lambda_{N} \in N_{\mathfrak{R}_{-}^{p}}\left(\widehat{g}^{N}\left(z_{N}\right)\right), \\
& \delta_{N} \in N_{\mathfrak{R}_{-}^{m}}\left(\widehat{H}^{N}\left(z_{N}\right) \circ \widehat{G}^{N}\left(z_{N}\right)-t_{N} e\right) \text {, } \\
& a_{N} \in N_{\mathfrak{R}_{+}^{m}}\left(\widehat{G}^{N}\left(z_{N}\right)\right), \\
& b_{N} \in N_{\Re_{+}^{m}}\left(\widehat{H}^{N}\left(z_{N}\right)\right), \quad v_{i}^{N}=\left(\delta_{N}\right)_{i} \widehat{G}_{i}^{N}\left(z_{N}\right) \text {, } \\
& u_{i}^{N}=\left(\delta_{N}\right)_{i} \widehat{H}_{i}^{N}\left(z_{N}\right), \quad i=1,2, \ldots, m,
\end{aligned}
$$

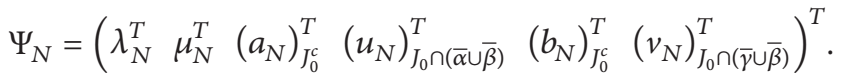

Next we show that $\Psi_{N}$ is almost surely bounded under the MPCC-MFCQ. We assume by contradiction that $\Psi_{N}$ is unbounded, then there exists a number sequence $\left\{\tau_{N}\right\} \searrow 0$ such that $\tau_{N} \Psi_{N} \rightarrow \bar{\Psi} \neq 0$, where

$$
\bar{\Psi}=\left(\begin{array}{llllll}
\bar{\lambda}^{T} & \bar{\mu}^{T} & \bar{a}_{J_{0}^{c}}^{T} & \bar{u}_{J_{0} \cap(\bar{\alpha} \cup \bar{\beta})}^{T} & \bar{b}_{J_{0}^{c}}^{T} & \bar{v}_{J_{0} \cap(\bar{\gamma} \cup \bar{\beta})}^{T}
\end{array}\right)^{T} .
$$

Since

$\frac{\widehat{H}_{i}^{N}\left(z_{N}\right)}{\widehat{G}_{i}^{N}\left(z_{N}\right)} \nabla \widehat{G}_{i}^{N}\left(z_{N}\right)^{T} \longrightarrow 0, \quad i \in J_{0} \cap \bar{\gamma}$ w.p.1 as $N \longrightarrow \infty$,

$\frac{\widehat{G}_{i}^{N}\left(z_{N}\right)}{\widehat{H}_{i}^{N}\left(z_{N}\right)} \nabla \widehat{H}_{i}^{N}\left(z_{N}\right)^{T} \longrightarrow 0, \quad i \in J_{0} \cap \bar{\alpha}$, w.p.1 as $N \longrightarrow \infty$

and by outer semicontinuousness of normal cone

$$
\begin{gathered}
\bar{a} \in N_{\Re_{+}^{m}}(\mathbb{E}[G(\bar{z}, \xi)]), \\
\bar{b} \in N_{\Re_{+}^{m}}(\mathbb{E}[H(\bar{z}, \xi)]), \\
\bar{u} \in N_{\Re_{-}^{m}}(\mathbb{E}[G(\bar{z}, \xi)] \mathbb{E}[H(\bar{z}, \xi)]), \\
\bar{v} \in N_{\Re_{-}^{m}}(\mathbb{E}[G(\bar{z}, \xi)] \mathbb{E}[H(\bar{z}, \xi)]) .
\end{gathered}
$$

Notice that $\bar{a}_{i}=0, i \in J_{0}^{c} \cap \bar{\gamma}$, and $\bar{b}_{i}=0, i \in J_{0}^{c} \cap \bar{\alpha}$; then by multiplying $\tau_{N}$ to both sides of (45) and taking limit, we have

$$
0=\left(\begin{array}{c}
\mathscr{J} \mathbb{E}[g(\bar{z}, \xi)] \\
\mathscr{J} \mathbb{E}[h(\bar{z}, \xi)] \\
\mathcal{J} \mathbb{E}[G(\bar{z}, \xi)]_{\bar{\alpha} \cup \bar{\beta}} \\
\mathscr{J} \mathbb{E}[H(\bar{z}, \xi)]_{\bar{\gamma} \cup \bar{\beta}}
\end{array}\right)^{T} \widehat{\Psi}
$$

with $\widehat{\Psi} \neq 0$, where

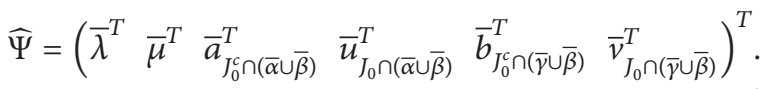

However, we know from MPCC-MFCQ that for any $A \in$ $\partial^{c} \min \{\mathbb{E}[G(\bar{z}, \xi)]$ and $\mathbb{E}[H(\bar{z}, \xi)]\}$

$$
\begin{array}{r}
0 \in \operatorname{int}\left\{\left(\begin{array}{c}
\mathbb{E}[g(\bar{z}, \xi)] \\
\mathbb{E}[h(\bar{z}, \xi)] \\
\min \{\mathbb{E}[G(\bar{z}, \xi)], \mathbb{E}[H(\bar{z}, \xi)]\}
\end{array}\right)\right. \\
\left.+\left(\begin{array}{c}
\mathscr{J} \mathbb{E}[g(\bar{z}, \xi)] \\
\mathscr{J} \mathbb{E}[h(\bar{z}, \xi)] \\
A
\end{array}\right) \Re^{n}-\left(\begin{array}{c}
\mathfrak{R}_{-}^{p} \\
\{0\}_{q} \\
\{0\}_{m}
\end{array}\right)\right\},
\end{array}
$$

which is called the generalized Robinson constraint qualification in [19]. Notice that for $A_{i} \in \partial^{c} \min \left\{\mathbb{E}\left[G_{i}(\bar{z}, \xi)\right]\right.$ and $\left.\mathbb{E}\left[H_{i}(\bar{z}, \xi)\right]\right\}$, there exists $k \in[0,1]$ such that

$$
A_{i}= \begin{cases}\nabla \mathbb{E}\left[G_{i}(\bar{z}, \xi)\right], & i \in \bar{\alpha} \\ \nabla \mathbb{E}\left[H_{i}(\bar{z}, \xi)\right], & i \in \bar{\gamma} \\ k \nabla \mathbb{E}\left[G_{i}(\bar{z}, \xi)\right]+(1-k) \nabla \mathbb{E}\left[H_{i}(\bar{z}, \xi)\right], & i \in \bar{\beta} .\end{cases}
$$

Then by dual form of generalized Robinson constraint qualification in Yen [19], we have for any $k \in[0,1]$

0

$$
\begin{aligned}
& =\left(\begin{array}{c}
\mathscr{J} \mathbb{E}[g(\bar{z}, \xi)] \\
\mathscr{J} \mathbb{E}[h(\bar{z}, \xi)] \\
\nabla \mathbb{E}\left[G_{i}(\bar{z}, \xi)\right]^{T}, i \in \bar{\alpha} \\
\nabla \mathbb{E}\left[H_{i}(\bar{z}, \xi)\right]^{T}, i \in \bar{\gamma} \\
k \nabla \mathbb{E}\left[G_{i}(\bar{z}, \xi)\right]^{T}+(1-k) \nabla \mathbb{E}\left[H_{i}(\bar{z}, \xi)\right]^{T}, i \in \bar{\beta} \\
\lambda \in N_{\mathfrak{R}_{-}^{p} \times\{0\}_{q} \times\{0\}_{m}}(0)
\end{array}\right)^{T} \lambda \\
& \Longrightarrow \lambda=0,
\end{aligned}
$$

which means that

$$
\begin{aligned}
& 0\left.=\left(\begin{array}{c}
\mathscr{g} \mathbb{E}[g(\bar{z}, \xi)] \\
\mathcal{J} \mathbb{E}[h(\bar{z}, \bar{\xi})] \\
\nabla \mathbb{E}\left[G_{i}(\bar{z}, \xi)\right]^{T}, i \in \bar{\alpha} \\
\nabla \mathbb{E}\left[H_{i}(\bar{z}, \xi)\right]^{T}, i \in \bar{\gamma} \\
\nabla \mathbb{E}\left[G_{i}(\bar{z}, \xi)\right]^{T}, i \in \bar{\beta} \\
\nabla \mathbb{E}\left[H_{i}(\bar{z}, \xi)\right]^{T}, i \in \bar{\beta}
\end{array}\right)\left(\begin{array}{c}
\mu^{h} \\
\mu^{g} \\
\mu_{\bar{\alpha}} \\
\mu_{\bar{\gamma}} \\
\mu^{G} \\
\mu^{H}
\end{array}\right)\right\} \\
& \mu_{i}^{G} \mu_{i}^{H} \geq 0, i \in \bar{\beta} \\
&=\left(\begin{array}{c}
\mu^{h} \\
\mu^{g} \\
\mu_{\bar{\alpha}} \\
\mu_{\bar{\gamma}} \\
\mu^{G} \\
\mu^{H}
\end{array}\right)=0 .
\end{aligned}
$$

That is, $\widehat{\Psi}$ in (50) is 0 . This contradicts the condition that $\widehat{\Psi} \neq 0$ and hence $\left\{\Psi_{N}\right\}$ is bounded. Without loss of generality, we assume $\Psi_{N} \rightarrow \widetilde{\Psi}$ w.p.1 as $N \rightarrow \infty$, where

$$
\widetilde{\Psi}=\left(\begin{array}{llllll}
\tilde{\lambda}^{T} & \widetilde{\mu}^{T} & \widetilde{a}_{J_{0}^{c}}^{T} & \widetilde{u}_{J_{0} \cap(\bar{\alpha} \cup \bar{\beta})}^{T} & \widetilde{b}_{J_{0}^{c}}^{T} & \widetilde{v}_{J_{0} \cap(\bar{\gamma} \cup \bar{\beta})}^{T}
\end{array}\right)^{T} .
$$


Notice that

$$
\begin{gathered}
\left(a_{N}\right)_{J_{0}^{c}} \longrightarrow \widetilde{a}_{J_{0}^{c}}, \quad\left(b_{N}\right)_{J_{0}^{c}} \longrightarrow \widetilde{b}_{J_{0}^{c}} \quad \text { w.p.1, } \\
\left(\delta_{N}\right)_{i} \widehat{H}_{i}^{N}\left(z_{N}\right) \longrightarrow \widetilde{u}_{i}, \quad i \in J_{0} \cap(\bar{\alpha} \cup \bar{\beta}), \\
\left(\delta_{N}\right)_{i} \widehat{G}_{i}^{N}\left(z_{N}\right) \longrightarrow \widetilde{v}_{i}, \quad i \in J_{0} \cap(\bar{\gamma} \cup \bar{\beta}) \text { w.p.1. }
\end{gathered}
$$

Then we know from (46) that for $i \in \bar{\beta}$, in the case when $i \in J_{0}^{c}, \tilde{a}_{i} \widetilde{b}_{i} \geq 0$ due to $\left(a_{N}\right)_{i} \leq 0$ and $\left(b_{N}\right)_{i} \leq 0$ for each $N$. In the case when $i \in J_{0}$, since

$$
\left(\delta_{N}\right)_{i} \widehat{H}_{i}^{N}\left(z_{N}\right)\left(\delta_{N}\right)_{i} \widehat{G}_{i}^{N}\left(z_{N}\right) \geq 0,
$$

we have $\tilde{u}_{i} \widetilde{v}_{i} \geq 0$. As a result, by Definition $7, \bar{z}$ is a $C$ stationary point. If $\widetilde{\Psi}_{i}=0$ for $i \in J_{0} \cap \bar{\beta}$, then we know from Definition 7 that $\bar{z}$ is a $S$-stationary point. The proof is completed.

Remark 18. For a deterministic MPCC problem, Scholtes [17] studied the properties of the limit point of a sequence of stationary points generated by the same regularization method under MPCC-LICQ. Notice that MPCC-MFCQ in Theorem 17 is weaker than MPCC-LICQ. Thus this theorem can be seen as an improvement of [17, Theorem 3.1] for solving SMPCC under weaker constraint qualification conditions.

\section{Existence and Exponential Convergence Rate}

In this section, we discuss the conditions ensuring existence and exponential convergence of stationary points of regularized SAA problem satisfying (40) when the sample size is sufficiently large.

We need the following lemma.

Lemma 19. Let $X \subseteq \mathfrak{R}^{n}$ be a compact set. Suppose Assumptions 10-12 hold. Then for any $\varepsilon>0$, there exist positive constants $C(\varepsilon)$ and $\beta(\varepsilon)$, independent of $N$, such that

$$
\operatorname{Prob}\left\{\sup _{z \in X}\left\|\nabla \widehat{f}^{N}(z)-\nabla \mathbb{E}[f(z, \xi(\omega))]\right\| \geq \varepsilon\right\} \leq C(\varepsilon) e^{-N \beta(\varepsilon)} .
$$

Proof. Under Assumptions 10-12, we know from [10, Theorem 7.65] that for each $i \in\{1,2, \ldots, n\}$, there exist positive constants $C_{i}(\varepsilon)$ and $\beta_{i}(\varepsilon)$, independent of $N$, such that

$$
\begin{aligned}
& \operatorname{Prob}\left\{\sup _{z \in X}\left\|\nabla \widehat{f}^{N}(z)_{i}-\nabla \mathbb{E}[f(z, \xi(\omega))]_{i}\right\| \geq \frac{\varepsilon}{n}\right\} \\
& \leq C_{i}(\varepsilon) e^{-N \beta_{i}(\varepsilon)},
\end{aligned}
$$

where $\nabla \widehat{f}^{N}(z)_{i}$ and $\nabla \mathbb{E}[f(z, \xi(\omega))]_{i}$ denote the $i$ th component of $\nabla \hat{f}^{N}(z)$ and $\nabla \mathbb{E}[f(z, \xi(\omega))]$, respectively. Therefore, we have

$$
\begin{aligned}
\operatorname{Prob} & \left\{\sup _{z \in X}\left\|\nabla \widehat{f}^{N}(z)-\nabla \mathbb{E}[f(z, \xi(\omega))]\right\| \geq \varepsilon\right\} \\
& \leq \sum_{i=1}^{n} \operatorname{Prob}\left\{\sup _{z \in X}\left\|\nabla \widehat{f}^{N}(z)_{i}-\nabla \mathbb{E}[f(z, \xi(\omega))]_{i}\right\| \geq \frac{\varepsilon}{n}\right\} \\
& \leq \sum_{i=1}^{n} C_{i}(\varepsilon) e^{-N \beta_{i}(\varepsilon)} \leq C(\varepsilon) e^{-N \beta(\varepsilon)},
\end{aligned}
$$

where $C(\varepsilon):=n \max \left\{C_{1}(\varepsilon), C_{2}(\varepsilon), \ldots, C_{n}(\varepsilon)\right\}$, and $\beta(\varepsilon):=$ $\min \left\{\beta_{1}(\varepsilon), \beta_{2}(\varepsilon), \ldots, \beta_{n}(\varepsilon)\right\}$.

We now state our existence and exponential convergence results. The proof relies on an application of Robinson's standard NLP stability theory in [20].

Theorem 20. Let $\bar{z}$ be a C-stationary point of SMPCC (1) and $\tau_{N} \searrow 0$. Suppose

(i) Assumptions 10-12 hold at $\bar{z}$,

(ii) MPCC-LICQ (Definition 6), MPCC-SSOSC (Definition 9), and ULSC (Definition 8) hold at $\bar{z}$.

Then we have that

(a) there exits $z_{N}$ satisfying stationary condition (40) of (3) w.p.1 for each $N$ when $N$ is sufficiently large and $z_{N} \rightarrow$ $\bar{z}$ w.p.1 as $N \rightarrow \infty$;

(b) the sequence $\left\{z_{N}\right\}$ in (a) satisfies that for every $\varepsilon>0$, there exist positive constants $C(\varepsilon)$ and $\beta(\varepsilon)$, independent of $N$, such that

$$
\operatorname{Prob}\left\{\left\|z_{N}-\bar{z}\right\| \geq \varepsilon\right\} \leq C(\varepsilon) e^{-N \beta(\varepsilon)}
$$

for $N$ sufficiently large.

Proof. Since $\bar{z}$ is a $C$-stationary point of SMPCC, then there exist vectors $\bar{\lambda} \in \mathfrak{R}^{\left|I_{g}\right|}, \bar{\mu} \in \mathfrak{R}^{q}, \bar{u} \in \mathfrak{R}^{|\bar{\alpha}|+|\bar{\beta}|}$, and $\bar{v} \in \mathfrak{R}^{|\bar{\beta}|+|\bar{\gamma}|}$ such that

$$
\begin{gathered}
G(\bar{z}, \bar{\lambda}, \bar{\mu}, \bar{u}, \bar{v})=0, \\
\bar{u}_{i} \bar{v}_{i} \geq 0, \quad i \in \bar{\beta},
\end{gathered}
$$

where

$$
G(z, \lambda, \mu, u, v)=\left[\begin{array}{c}
\nabla_{z} L(z, \lambda, \mu, u, v) \\
\mathbb{E}\left[g_{I_{g}}(z, \xi)\right] \\
\mathbb{E}[h(z, \xi)] \\
\mathbb{E}\left[G_{\bar{\alpha} \cup \bar{\beta}}(z, \xi)\right] \\
\mathbb{E}\left[H_{\bar{\beta} \cup \bar{\gamma}}(z, \xi)\right]
\end{array}\right]
$$


with

$$
\begin{aligned}
L(z, \lambda, \mu, u, v) \\
=\mathbb{E}[f(z, \xi)]+\sum_{i \in I_{g}} \lambda_{i} \mathbb{E}\left[g_{i}(z, \xi)\right] \\
\quad+\sum_{i=1}^{q} \mu_{i} \mathbb{E}\left[h_{i}(z, \xi)\right] \\
\quad-\sum_{i \in \bar{\alpha} \cup \bar{\beta}} u_{i} \mathbb{E}\left[G_{i}(z, \xi)\right]-\sum_{i \in \bar{\beta} \cup \bar{\gamma}} v_{i} \mathbb{E}\left[H_{i}(z, \xi)\right] .
\end{aligned}
$$

Notice that (63) can be seen as a KKT condition of the following NLP problem:

$$
\begin{array}{ll}
\min & \mathbb{E}[f(z, \xi)] \\
\text { s.t. } & \mathbb{E}\left[g_{i}(z, \xi)\right]=0, \quad i \in I_{g}, \\
& \mathbb{E}[h(z, \xi)]=0, \\
& \mathbb{E}\left[G_{i}(z, \xi)\right]=0, \quad i \in \bar{\alpha} \cup \bar{\beta}, \\
& \mathbb{E}\left[H_{i}(z, \xi)\right]=0, \quad i \in \bar{\beta} \cup \bar{\gamma} .
\end{array}
$$

The MPCC-SSOSC ensures the strong second-order sufficient condition for NLP problem (67), which, under MPCCLICQ, implies the stability of (67) in the sense of Robinson [20]. Hence, there exist positive numbers $\varepsilon, \delta$, and $c$ such that for every $p \in \mathbb{B}(0, \varepsilon)$, the mapping $\Sigma(p)=\{\theta \in$ $\left.\mathfrak{R}^{n+\left|I_{g}\right|+q+|\bar{\alpha}|+2|\bar{\beta}|+|\bar{\gamma}|} \mid 0 \in G(\theta)+p, \theta=(z, \lambda, \mu, u, v)\right\}$ has only one solution $\theta(p):=(z(p), \lambda(p), \mu(p), u(p), v(p)) \epsilon$ $\mathbb{B}(\bar{\theta}, \delta)$ with $\bar{\theta}=(\bar{z}, \bar{\lambda}, \bar{\mu}, \bar{u}, \bar{v})=z(0)$ and the mapping $\theta(\cdot): \mathbb{B}(0, \varepsilon) \rightarrow \mathbb{B}(\bar{\theta}, \delta)$ satisfying

$$
\left\|\theta(p)-\theta\left(p^{\prime}\right)\right\| \leq c\left\|p-p^{\prime}\right\| \quad \text { for any } p, p^{\prime} \in \mathbb{B}(0, \varepsilon) .
$$

Since ULSC holds at $\bar{z}$ and $\bar{z}$ is a $C$-stationary point, we have $\bar{u}_{i} \bar{v}_{i}>0$ for $i \in \beta$, which means that for sufficiently small $\delta>0$ and any $\theta \in \mathbb{B}(\bar{\theta}, \delta), v_{i} / u_{i}>0$, and $u_{i} / v_{i}>0, i \in \beta$. Let

$$
Q^{N}(\theta)=\left[\begin{array}{c}
Q_{1}^{N}(\theta) \\
\mathbb{E}[h(z, \xi)]-\widehat{h}^{N}(z) \\
\mathbb{E}\left[g_{I_{g}}(z, \xi)\right]-\widehat{g}_{I_{g}}^{N}(z) \\
\mathbb{E}\left[G_{i}(z, \xi)\right]-\widehat{G}^{N}(z)+a_{N}, i \in \alpha \cup \bar{\beta} \\
\mathbb{E}\left[H_{i}(z, \xi)\right]-\widehat{H}^{N}(z)+b_{N}, i \in \bar{\gamma} \cup \bar{\beta}
\end{array}\right],
$$

where

$$
\begin{aligned}
Q_{1}^{N}( & \theta) \\
= & \nabla \widehat{f}_{N}(z)-\nabla \mathbb{E}[f(z, \xi)] \\
& +\sum_{i \in I_{g}} \lambda_{i}\left[\nabla \mathbb{E}\left[g_{i}(z, \xi)\right]-\nabla \widehat{g}_{i}^{N}(z)\right] \\
& +\sum_{i=1}^{q} \mu_{i}\left[\nabla \mathbb{E}\left[h_{i}(z, \xi)\right]-\nabla \widehat{h}_{i}^{N}(z)\right] \\
& -\sum_{i \in \alpha \cup \bar{\gamma}} u_{i}\left[\nabla \mathbb{E}\left[G_{i}(z, \xi)\right]-\nabla \widehat{G}_{i}^{N}(z)\right] \\
& -\sum_{i \in \bar{\beta} \cup \bar{\gamma}} v_{i}\left[\nabla \mathbb{E}\left[H_{i}(z, \xi)\right]-\nabla \widehat{H}_{i}^{N}(z)\right] \\
& +\sum_{i \in \gamma} u_{i} \frac{t_{N}}{\left(\widehat{G}_{i}^{N}(z)\right)^{2}} \nabla \widehat{G}_{i}^{N}(z)+\sum_{i \in \alpha} v_{i} \frac{t_{N}}{\left(\widehat{H}_{i}^{N}(z)\right)^{2}} \nabla \widehat{H}_{i}^{N}(z), \\
& a_{N}=\sqrt{\frac{v_{i}}{u_{i}} t_{N}}, \quad b_{N}=\sqrt{\frac{u_{i}}{v_{i}} t_{N} .}
\end{aligned}
$$

For sufficiently small $\delta>0$ and sufficiently large $N, \widehat{H}_{i}^{N}(z)>$ $0, i \in \alpha$, and $\widehat{G}_{i}^{N}(z)>0, i \in \gamma$. Then by Lemma 13, we have that

$$
\begin{aligned}
& \sup _{\theta \in \mathbb{B}(\bar{\theta}, \delta)}\left\|\sum_{i \in \gamma} u_{i} \frac{t_{N}}{\left(\widehat{G}_{i}^{N}(z)\right)^{2}} \nabla \widehat{G}_{i}^{N}(z)\right\| \longrightarrow 0, \\
& \sup _{\theta \in \mathbb{B}(\bar{\theta}, \delta)}\left\|\sum_{i \in \alpha} v_{i} \frac{t_{N}}{\left(\widehat{H}_{i}^{N}(z)\right)^{2}} \nabla \widehat{H}_{i}^{N}(z)\right\| \longrightarrow 0
\end{aligned}
$$

w.p.1 as $N \rightarrow \infty$. By the Uniform Laws of Large Numbers, we have

$$
\sup _{\theta \in \mathbb{B}(\bar{\theta}, \delta)}\left\|\nabla \hat{f}^{N}(z)-\nabla \mathbb{E}[f(z, \xi)]\right\| \longrightarrow 0,
$$

$$
\begin{gathered}
\sup _{\theta \in \mathbb{B}(\bar{\theta}, \delta)}\left\|\lambda_{i}\left(\nabla \widehat{\mathfrak{g}}_{i}^{N}(z)-\nabla \mathbb{E}\left[g_{i}(z, \xi)\right]\right)\right\| \longrightarrow 0, \quad i \in I_{g}, \\
\sup _{\theta \in \mathbb{B}(\bar{\theta}, \delta)}\left\|\mu_{i}\left(\nabla \widehat{h}_{i}^{N}(z)-\nabla \mathbb{E}\left[h_{i}(z, \xi)\right]\right)\right\| \longrightarrow 0, \quad i=1,2, \ldots, q, \\
\sup _{\theta \in \mathbb{B}(\bar{\theta}, \delta)}\left\|u_{i}\left(\nabla \widehat{G}_{i}^{N}(z)-\nabla \mathbb{E}\left[G_{i}(z, \xi)\right]\right)\right\| \longrightarrow 0, \quad i \in \bar{\alpha} \cup \bar{\beta},
\end{gathered}
$$

$$
\sup _{\theta \in \mathbb{B}(\bar{\theta}, \delta)}\left\|v_{i}\left(\nabla \widehat{H}_{i}^{N}(z)-\nabla \mathbb{E}\left[H_{i}(z, \xi)\right]\right)\right\| \longrightarrow 0, \quad i \in \bar{\beta} \cup \bar{\gamma}
$$

w.p.1 as $N \rightarrow \infty$. As a result, combining (70)-(73), we obtain that for $\varepsilon>0$, when $N$ is sufficiently large,

$$
\left\|Q_{1}^{N}\right\|_{\delta}=\sup _{\theta \in \mathbb{B}(\bar{\theta}, \delta)}\left\|Q_{1}^{N}(\theta)\right\|<\varepsilon \quad \text { w.p.1. }
$$


In addition, we know from Uniform Laws of Large Numbers that

$$
\sup _{\theta \in \mathbb{B}(\bar{\theta}, \delta)}\left\|\varrho_{N}(z)-\mathbb{E}\left[\varrho_{N}(z)\right]\right\| \longrightarrow 0 \quad \text { w.p.1 as } N \longrightarrow \infty,
$$

which implies that for above $\varepsilon>0$, when $N$ is sufficiently large,

$$
\sup _{\theta \in \mathbb{B}(\bar{\theta}, \delta)}\left\|\varrho_{N}(z)-\mathbb{E}\left[\varrho_{N}(z)\right]\right\|<\varepsilon \quad \text { w.p.1, }
$$

where $\varrho_{N}(z)$ is any element in $\left\{\widehat{g}_{i}^{N}(z), i \in I_{g}, \widehat{h}_{i}^{N}(z), i=\right.$ $1,2, \ldots, q, \widehat{H}_{i}^{N}(z), i \in \bar{\beta} \cup \bar{\gamma}, \widehat{G}_{i}^{N}(z)$, and $i \in \bar{\beta} \cup \bar{\alpha}$. Hence, we know from (69), (74), and (76) that for above $\varepsilon>0$ when $N$ is sufficiently large,

$$
\left\|Q^{N}\right\|_{\delta}=\sup _{\theta \in \mathbb{B}(\bar{\theta}, \delta)}\left\|Q^{N}(\theta)\right\|<\varepsilon \quad \text { w.p.1. }
$$

Applying the Brouwer's fixed point theorem to the mapping $\theta\left(Q^{N}(\cdot)\right): \mathbb{B}(\bar{\theta}, \delta) \rightarrow \mathbb{B}(\bar{\theta}, \delta)$, where $\theta(\cdot)$ is defined as in (68), we conclude that there is at least one fixed point $\theta_{N}=\left(z_{N}, \lambda_{N}, \mu_{N}, u_{N}, v_{N}\right) \in \Re^{n+\left|I_{g}\right|+q+|\bar{\alpha}|+2|\bar{\beta}|+|\bar{\gamma}|}$ such that $\theta_{N}=\theta\left(Q^{N}\left(\theta_{N}\right)\right)$ w.p.1. Therefore, when $N$ is sufficiently large, there exists $\theta_{N} \in \mathbb{B}(\bar{\theta}, \delta)$ w.p.1 such that $0 \in G\left(\theta_{N}\right)+Q^{N}\left(\theta_{N}\right)$ w.p.1, namely,

$$
\begin{aligned}
0 \in \nabla & \widehat{f}^{N}\left(z_{N}\right)+\sum_{i \in I_{g}}\left(\lambda_{N}\right)_{i} \nabla \widehat{g}_{i}^{N}\left(z_{N}\right)+\sum_{i=1}^{q}\left(\mu_{N}\right)_{i} \nabla \widehat{h}_{i}^{N}\left(z_{N}\right) \\
& -\sum_{i \in \bar{\alpha}}\left[\left(u_{N}\right)_{i} \nabla \widehat{G}_{i}^{N}\left(z_{N}\right)+\left(u_{N}\right)_{i} \frac{t_{N}}{\left(H_{i}^{N}(z)\right)^{2}} \nabla \widehat{H}_{i}^{N}\left(z_{N}\right)\right] \\
& -\sum_{i \in \bar{\gamma}}\left[\left(v_{N}\right)_{i} \nabla \widehat{H}_{i}^{N}\left(z_{N}\right)+\left(v_{N}\right)_{i} \frac{t_{N}}{\left(\widehat{G}_{i}^{N}\left(z_{N}\right)\right)^{2}} \nabla \widehat{G}_{i}^{N}\left(z_{N}\right)\right] \\
& -\sum_{i \in \bar{\beta}}\left[\left(u_{N}\right)_{i} \nabla \widehat{G}_{i}^{N}\left(z_{N}\right)+\left(v_{N}\right)_{i} \nabla \widehat{H}_{i}^{N}\left(z_{N}\right)\right] \quad \text { w.p.1 }
\end{aligned}
$$

with

$$
\begin{gathered}
\widehat{h}^{N}\left(z_{N}\right)=0, \quad \widehat{g}_{I_{g}}^{N}\left(z_{N}\right)=0, \\
\widehat{G}_{i}^{N}\left(z_{N}\right) \widehat{H}_{i}^{N}\left(z_{N}\right)=t_{N}, \quad i \in \alpha \cup \gamma, \\
\widehat{G}_{i}^{N}\left(z_{N}\right)=\sqrt{\frac{\left(v_{N}\right)_{i}}{\left(u_{N}\right)_{i}} t_{N},} \\
\widehat{H}_{i}^{N}\left(z_{N}\right)=\sqrt{\frac{\left(u_{N}\right)_{i}}{\left(v_{N}\right)_{i}} t_{N},} \quad i \in \beta .
\end{gathered}
$$

Moreover, combining (68) and (77), we obtain

$$
\theta_{N} \longrightarrow \bar{\theta} \quad \text { w.p.1 as } N \longrightarrow \infty \text {. }
$$

Let

$$
\left(\delta_{N}\right)_{i}= \begin{cases}\frac{\left(u_{N}\right)_{i}}{\widehat{H}_{i}^{N}\left(z_{N}\right)}, & i \in \bar{\alpha}, \\ \sqrt{\frac{\left(u_{N}\right)_{i}\left(v_{N}\right)_{i}}{t_{N}},} & i \in \bar{\beta}, \\ \frac{\left(v_{N}\right)_{i}}{\widehat{G}_{i}^{N}\left(z_{N}\right)}, & i \in \bar{\gamma},\end{cases}
$$

then we have from (78) that $z_{N}$ is almost surely a stationary point of (3) and $\left(\lambda_{N}, \mu_{N}, \delta_{N}\right)$ is the corresponding multiplier. Furthermore, by (80), we have $z_{N} \rightarrow \bar{z}$ w.p.1 as $N \rightarrow \infty$. The proof of part (a) is completed.

Under condition (ii), we know from (68) and (77) that there exist $\kappa>0$ and $\delta>0$ such that

$$
\left\|z_{N}-\bar{z}\right\| \leq\left\|\theta_{N}-\bar{\theta}\right\| \leq c\left\|Q^{N}\left(z_{N}\right)\right\| \leq c \max _{\theta \in \mathbb{B}(\bar{\theta}, \delta)}\left\|Q^{N}(\theta)\right\| .
$$

For $\varepsilon>0$, combining (70)-(73), we obtain that when $N$ is large enough

$$
\max _{\theta \in \mathbb{B}(\bar{\theta}, \delta)}\left\|Q_{1}^{N}(z)\right\| \leq \max _{z \in \mathbb{B}(\bar{z}, \delta)}\left\|\nabla \widehat{f}_{N}(z)-\nabla \mathbb{E}[f(z, \xi)]\right\|+\frac{\varepsilon}{4 c},
$$

which, by (76), means that when $N$ is large enough

$$
\max _{\theta \in \mathbb{B}(\bar{\theta}, \delta)}\left\|Q^{N}(z)\right\| \leq \max _{z \in \mathbb{B}(\bar{z}, \delta)}\left\|\nabla \widehat{f}_{N}(z)-\nabla \mathbb{E}[f(z, \xi)]\right\|+\frac{\varepsilon}{2 c} .
$$

According to Lemma 19, there exist $C(\varepsilon)>0$ and $\beta(\varepsilon)>0$, independent of $N$, such that

$$
\begin{aligned}
\operatorname{Prob} & \left\{\max _{z \in \mathbb{B}(\bar{z}, \delta)}\left\|\nabla \widehat{f}_{N}(z)-\nabla \mathbb{E}[f(z, \xi(\omega))]\right\| \geq \varepsilon\right\} \\
& \leq C(\varepsilon) e^{-N \beta(\varepsilon)},
\end{aligned}
$$

when $N$ is large enough. As a result, the conclusion of (b) follows from (82) and (84).

\section{Numerical Results}

In this section, we present some preliminary numerical results obtained by the regularization SAA method. Our numerical experiments are carried out in MATLAB 7.1 running on a PC with Intel Pentium $\mathrm{M}$ of $1.60 \mathrm{GHz} \mathrm{CPU}$ and our tests are focused on different values of the regularization parameter $\tau$ and sample size $N$.

To see the performance of the regularization SAA method, we have also carried out tests for the smoothing SAA method [6] for (6.3) which incorporates a smoothing NCP scheme based on the following Chen-Harker-Kanzow-Smale (CHKS) smoothing function: 
TABLE 1: The computational results for Example 1.

\begin{tabular}{lccccc}
\hline Methods & $N$ & $\tau$ & $z_{N}$ & Obj & Iter \\
\hline REG & $10^{3}$ & $5 \times 10^{-1}$ & $(0.8119,0.8064,0.8144,0.3960,0.3951,0.3868)$ & 0.5435 & 5 \\
CHKS & $10^{3}$ & $5 \times 10^{-1}$ & $(0.6503,0.6444,0.6542,0.5947,0.5868,0.5784)$ & 1.3664 & 6 \\
\hline REG & $10^{4}$ & $5 \times 10^{-2}$ & $(0.7963,0.8029,0.8029,0.4081,0.3974,0.4004)$ & 0.5921 & 3 \\
CHKS & $10^{4}$ & $5 \times 10^{-2}$ & $(0.7937,0.8004,0.7996,0.4109,0.4004,0.4030)$ & 0.6021 & 5 \\
\hline REG & $10^{5}$ & $5 \times 10^{-3}$ & $(0.7993,0.8000,0.8006,0.4016,0.3999,0.4001)$ & 0.6008 & 3 \\
CHKS & $10^{5}$ & $5 \times 10^{-3}$ & $(0.7993,0.8001,0.8004,0.4017,0.4000,0.4000)$ & 0.6009 & 4 \\
\hline
\end{tabular}

$$
\phi_{\tau}(a, b)=\frac{1}{2}\left(a+b-\sqrt{(a-b)^{2}+4 \tau^{2}}\right)
$$

and compare the test results.

In our experiments, we employed the random number generator unifrnd, exprnd, and normrnd in MATLAB 7.1 to generate independently and identically distributed random samples $\left\{\xi^{1}, \xi^{2}, \ldots, \xi^{N}\right\}$. We solved problem (3) with $N$ and $\tau$ by the solver fmincon in MATLAB 7.1 to obtain the approximated optimal solution $z_{N}$. Throughout the tests, we recorded number of iterations of fmincon (Iter) and the values of the objective function at $z_{N}(\mathrm{Obj})$ and these quantities are displayed in the tables of test results.

In the tables below, "REG" and "CHKS" denote regularization SAA method and the smoothing SAA method based on the CHKS smoothing function, respectively.

The examples below varied from examples in Shapiro and $\mathrm{Xu}[6]$.

\section{Example 1. Consider}

$$
\begin{aligned}
\min f(z)=\mathbb{E} & {\left[\left(z_{1}-1\right)^{2}+\left(z_{2}-1\right)^{2}\right.} \\
& +\left(z_{3}-1\right)^{2}+0.5 z_{4}^{2} \xi_{1} \\
& \left.+z_{5}^{2}+z_{6}^{2} \xi_{3}+2 \xi_{2}-1\right]
\end{aligned}
$$

s.t. $\quad 0 \leq \mathbb{E}[G(z, \xi)] \perp \mathbb{E}[H(z, \xi)] \geq 0$,

where

$$
\begin{aligned}
& G(z, \xi)=\left(z_{4}-\frac{1}{2} z_{1}+\xi_{3}-1, z_{5}-\xi_{2} z_{2}, z_{6}-\frac{1}{4} \xi_{1} z_{3}\right)^{T}, \\
& H(z, \xi)=\left(z_{4}-z_{1}+0.25 \xi_{1}, z_{5}-z_{2}+\xi_{2}, z_{6}-z_{3}+0.5 \xi_{3}\right)^{T},
\end{aligned}
$$

$\xi=\left(\xi_{1}, \xi_{2}, \xi_{3}\right), \xi_{1}, \xi_{2}, \xi_{3}$ are independent random variables; $\xi_{1}$ has an exponential distribution $\operatorname{EXP}(\lambda=0.5) ; \xi_{2}$ has an uniform distribution on $[0,1]$; and $\xi_{3}$ has a normal distribution $\mathcal{N}\left(\mu, \sigma^{2}\right)$ with $\mu=1$ and $\sigma=0.1$. The constraint here, which is a complementarity problem, has a solution $z=\left(z_{1}, z_{2}, z_{3}, z_{4}, z_{5}, z_{6}\right)$, where

$$
z_{i+3}-\frac{1}{2} z_{i}= \begin{cases}\frac{1}{2} z_{i}-1, & \text { if } z_{i} \geq 2 \\ 0, & \text { otherwise }\end{cases}
$$

for $i=1,2,3$. Therefore, substituting above $\left(z_{3}, z_{4}, z_{5}\right)$ into the objective function, we obtain that $(0.8,0.8,0.8,0.4,0.4$,
0.4 ) is the exact optimal solution and 0.2 is the optimal value. The test results are presented in Table 1.

Example 2. Consider

$$
\begin{aligned}
& \min \quad f(z)=\mathbb{E}[ 2\left(z_{1}-2\right)^{2}+2 z_{2}^{2} \\
&+\left(z_{3}-3\right)^{2} \xi_{2}+z_{4}^{2} \xi_{2} \\
&\left.+z_{5}^{2} \xi_{1}+z_{6}^{2} \xi_{3}\right], \\
& \text { s.t. } \quad 0 \leq \mathbb{E}[G(z, \xi)] \perp \mathbb{E}[H(z, \xi)] \geq 0,
\end{aligned}
$$

where $G(z, \xi)=\left(z_{4}-2 z_{1} \xi_{1}+\xi_{2}-\xi_{1}, z_{5}+0.5 z_{2}^{2} \xi_{2}-\xi_{3}, z_{6}-\right.$ $\left.z_{3} \xi_{3}+\xi_{3}\right)^{T}$ are $H(z, \xi)=\left(z_{1}, z_{2}, z_{3}\right), \xi=\left(\xi_{1}, \xi_{2}, \xi_{3}\right), \xi_{1}$, $\xi_{2}, \xi_{3}$ are independent random variables; $\xi_{1}$ has a normal distribution $\mathcal{N}\left(\mu, \sigma^{2}\right)$ with $\mu=0.5$ and $\sigma=0.1, \xi_{2}$ an exponential distribution $\operatorname{EXP}(\lambda=0.5)$; and $\xi_{3}$ has a uniform distribution on $[0,2]$. The constraint has a solution $z=$ $\left(z_{1}, z_{2}, z_{3}, z_{4}, z_{5}, z_{6}\right)$, where

$$
\begin{gathered}
z_{4}= \begin{cases}z_{1}-1.5, & \text { if } z_{1} \geq 1.5, \\
0, & \text { otherwise, }\end{cases} \\
z_{5}= \begin{cases}1-z_{2}^{2}, & \text { if }-1 \leq x_{2} \leq 1, \\
0, & \text { otherwise, }\end{cases} \\
z_{6}= \begin{cases}2 z_{3}-2, & \text { if } x_{3} \geq 1, \\
0, & \text { otherwise. }\end{cases}
\end{gathered}
$$

Therefore, substituting the above $\left(z_{4}, z_{5}, z_{6}\right)$ into the objective function, we obtain that $(1.75,0,0.5,0.25,1,0)$ is the exact optimal solution and 0.75 is the optimal value. The test results are displayed in Table 2.

Our preliminary numerical results shown in Tables 1 and 2 reveal that our proposed method yields a reasonable solution of the problems considered. To compare with the smoothing SAA method, the regularization SAA method may need fewer iteration numbers.

\section{Conclusion}

In this paper, we focus on detailed analysis of convergence of a regularization SAA method for SMPCC (1). Almost sure convergence of optimal solutions of the regularized SAA problem is established by the notion of epiconvergence in variational analysis. We improve a convergence result established by 
TABLE 2: The computational results for Example 2.

\begin{tabular}{lccccc}
\hline Methods & $N$ & $\tau$ & $z_{N}$ & Obj & Iter \\
\hline REG & $10^{3}$ & $5 \times 10^{-1}$ & $(1.7521,0.0002,0.5000,0.2485,0.9800,0.0001)$ & 0.7257 & 7 \\
CHKS & $10^{3}$ & $5 \times 10^{-1}$ & $(1.6621,0.0000,0.4614,0.5857,1.1901,0.3015)$ & 1.7127 & 10 \\
\hline REG & $10^{4}$ & $5 \times 10^{-2}$ & $(1.7502,0.0001,0.4999,0.2498,0.9990,0.0000)$ & 0.7485 & 8 \\
CHKS & $10^{4}$ & $5 \times 10^{-2}$ & $(1.7500,0.0000,0.5000,0.2593,1.0015,0.0050)$ & 0.7609 & 11 \\
\hline REG & $10^{5}$ & $5 \times 10^{-3}$ & $(1.7498,0.0000,0.5000,0.2504,0.9999,0.0000)$ & 0.7503 & 12 \\
CHKS & $10^{5}$ & $5 \times 10^{-3}$ & $(1.7498,0.0000,0.5000,0.2505,0.9999,0.0000)$ & 0.7504 \\
\hline
\end{tabular}

Scholtes [17] on a regularization method for a deterministic MPCC under weaker constraint qualifications. Moreover, the exponential convergence rate of the sequence of KarashKuhn-Tucker points generated from the regularized SAA problem is obtained through an application of Robinson's stability theory.

\section{Conflict of Interests}

The authors declare that they have no financial and personal relationships with other people or organizations that can inappropriately influence the work; there is no professional or other personal interest of any nature or kind in any product, service, and/or company that could be construed as influencing the position presented in, or the review of, the paper.

\section{Acknowledgments}

The authors are supported by the National Natural Science Foundation of China under Project no. 11201210 and no. 11171138 and Scientific Research Fund of Liaoning Provincial Education Department under Project no. L2012385.

\section{References}

[1] Z.-Q. Luo, J.-S. Pang, and D. Ralph, Mathematical Programs with Equilibrium Constraints, Cambridge University Press, Cambridge, UK, 1996.

[2] J. V. Outrata, "Mathematical programs with equilibrium constraints: theory and numericalmethods," in Nonsmooth Mechanics of Solids, J. Haslinger and G. E. Stavroulakis, Eds., vol. 485 of CISM Courses and Lecture Notes, pp. 221-274, Springer, New York, 2006.

[3] S. I. Birbil, G. Gürkan, and O. Listeş, "Solving stochastic mathematical programs with complementarity constraints using simulation," Mathematics of Operations Research, vol. 31, no. 4, pp. 739-760, 2006.

[4] M. Patriksson, "On the applicability and solution of bilevel optimization models in transportation science: a study on the existence, stability and computation of optimal solutions to stochastic mathematical programs with equilibrium constraints," Transportation Research B, vol. 42, pp. 843-860, 2008.

[5] H. Xu, "An implicit programming approach for a class of stochastic mathematical programs with complementarity constraints," SIAM Journal on Optimization, vol. 16, no. 3, pp. 670696, 2006.
[6] A. Shapiro and H. Xu, "Stochastic mathematical programs with equilibrium constraints, modelling and sample average approximation," Optimization, vol. 57, no. 3, pp. 395-418, 2008.

[7] M. Patriksson and L. Wynter, "Stochastic mathematical programs with equilibrium constraints," Operations Research Letters, vol. 25, no. 4, pp. 159-167, 1999.

[8] S. M. Robinson, "Analysis of sample-path optimization," Mathematics of Operations Research, vol. 21, no. 3, pp. 513-528, 1996.

[9] H. Jiang and H. Xu, "Stochastic approximation approaches to the stochastic variational inequality problem," IEEE Transactions on Automatic Control, vol. 53, no. 6, pp. 1462-1475, 2008.

[10] A. Shapiro, D. Dentcheva, and A. Ruszczyński, Lectures on Stochastic Programming: Modeling and Theory, SIAM, Philadelphia, Pa, USA, 2009.

[11] E. L. Plambeck, B.-R. Fu, S. M. Robinson, and R. Suri, "Samplepath optimization of convex stochastic performance functions," Mathematical Programming, vol. 75, no. 2, pp. 137-176, 1996.

[12] A. Shapiro and T. Homem-de-Mello, "On the rate of convergence of optimal solutions of Monte Carlo approximations of stochastic programs," SIAM Journal on Optimization, vol. 11, no. 1, pp. 70-86, 2000.

[13] J. Linderoth, A. Shapiro, and S. Wright, "The empirical behavior of sampling methods for stochastic programming," Annals of Operations Research, vol. 142, pp. 215-241, 2006.

[14] J. Zhang, L.-w. Zhang, and L.-p. Pang, "On the convergence of coderivative of SAA solution mapping for a parametric stochastic variational inequality," Set-Valued and Variational Analysis, vol. 20, no. 1, pp. 75-109, 2012.

[15] J. Zhang, L.-w. Zhang, and S. Lin, "A class of smoothing SAA methods for a stochastic mathematical program with complementarity constraints," Journal of Mathematical Analysis and Applications, vol. 387, no. 1, pp. 201-220, 2012.

[16] H. Scheel and S. Scholtes, "Mathematical programs with complementarity constraints: stationarity, optimality, and sensitivity," Mathematics of Operations Research, vol. 25, no. 1, pp. 1-22, 2000.

[17] S. Scholtes, "Convergence properties of a regularization scheme for mathematical programs with complementarity constraints," SIAM Journal on Optimization, vol. 11, no. 4, pp. 918-936, 2001.

[18] R. T. Rockafellar and R. J.-B. Wets, Variational Analysis, vol. 317, Springer-Verlag, Berlin, Germany, 1998.

[19] N. D. Yen, "Stability of the solution set of perturbed nonsmooth inequality systems and application," Journal of Optimization Theory and Applications, vol. 93, no. 1, pp. 199-225, 1997.

[20] S. M. Robinson, "Strongly regular generalized equations," Mathematics of Operations Research, vol. 5, no. 1, pp. 43-62, 1980. 


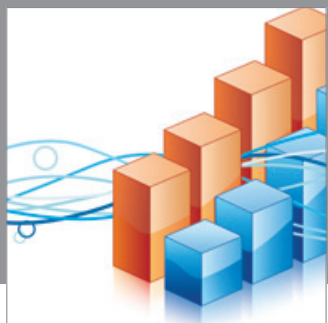

Advances in

Operations Research

mansans

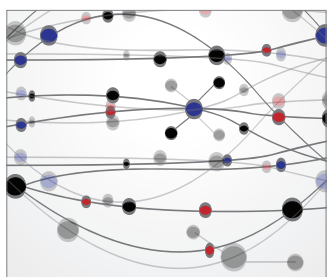

The Scientific World Journal
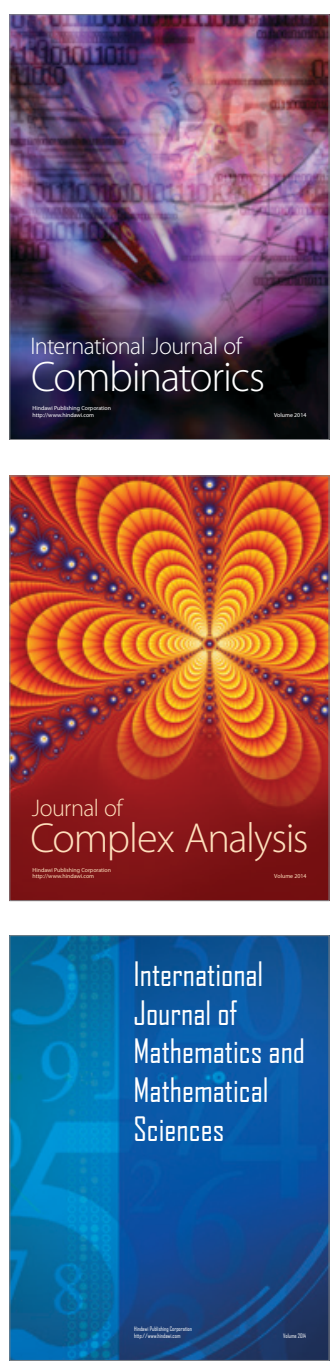
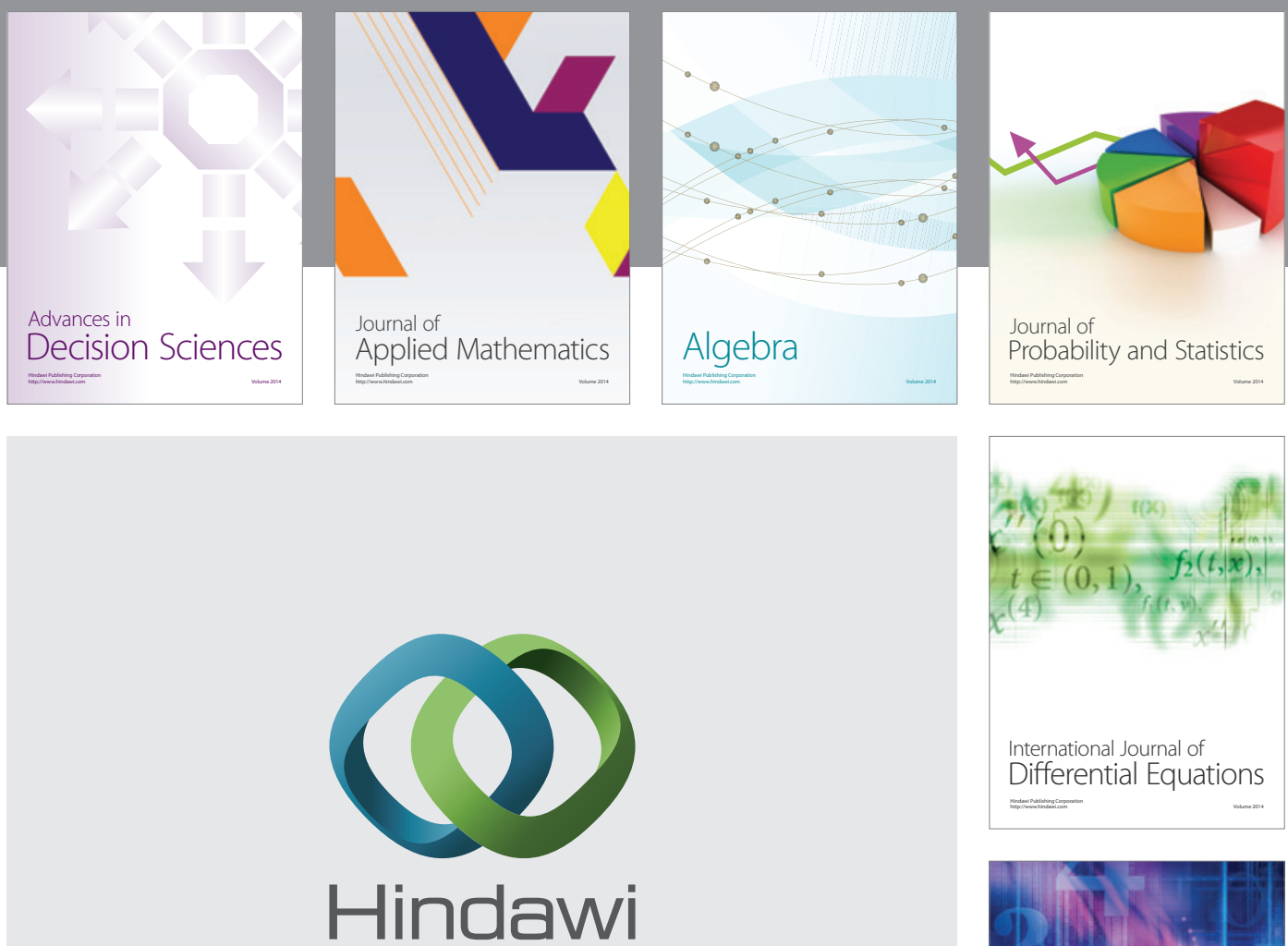

Submit your manuscripts at http://www.hindawi.com
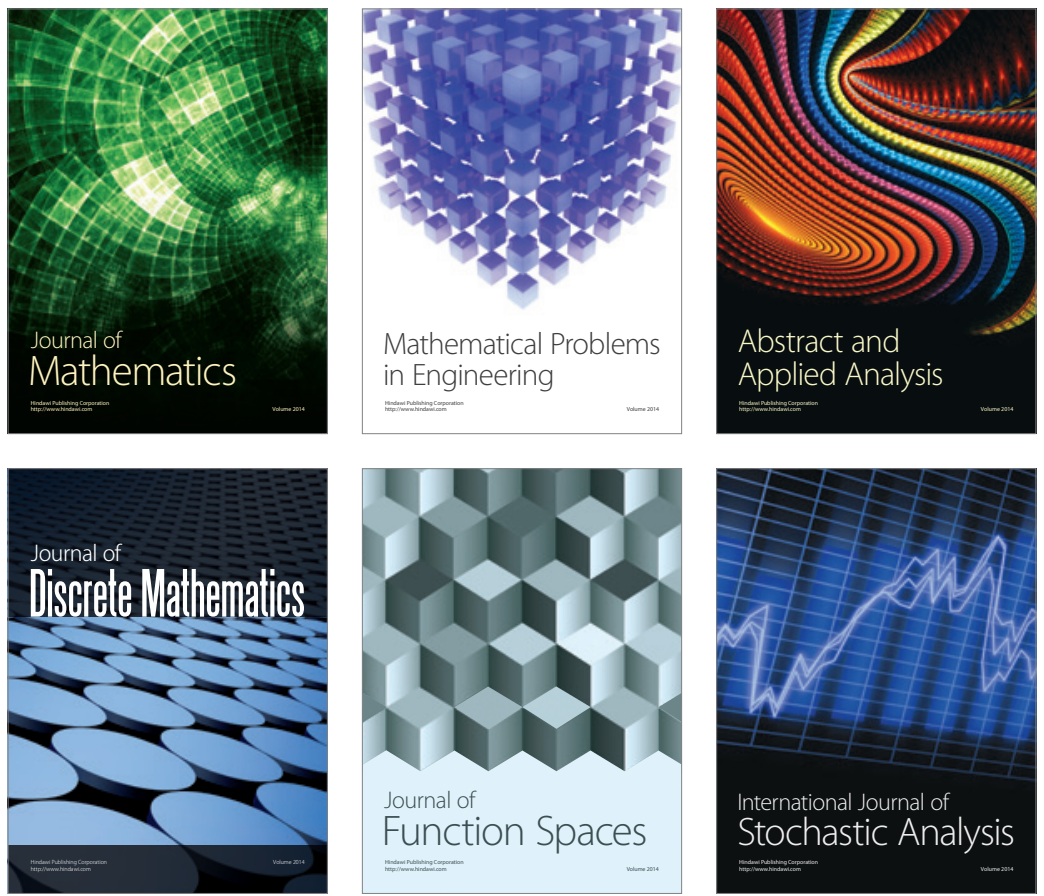

Journal of

Function Spaces

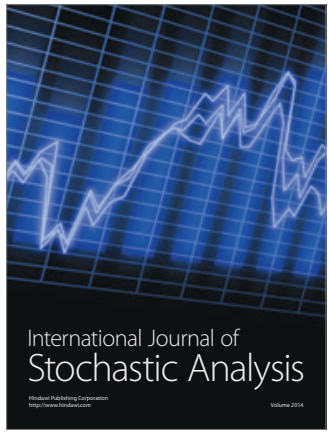

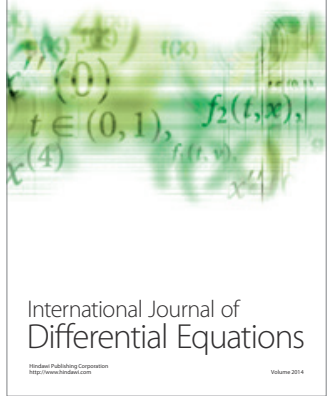
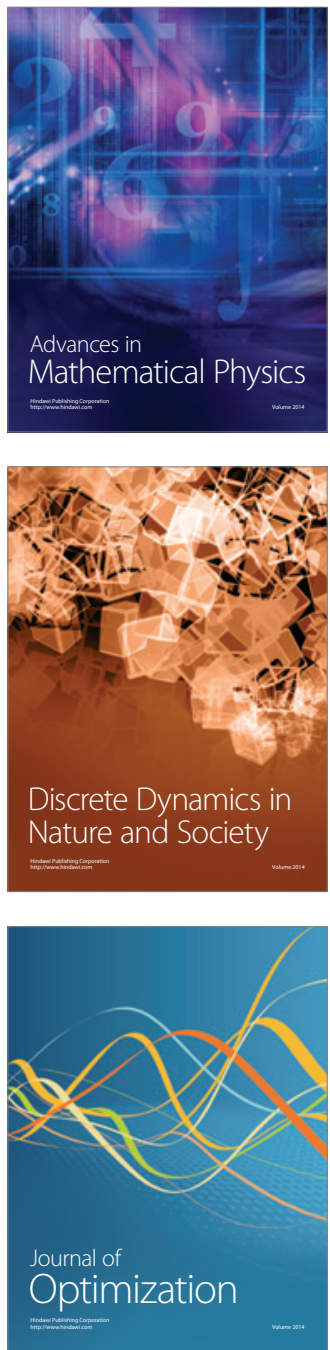